\title{
Efficient Allyl to Propenyl Isomerization in Functionally Diverse Compounds with a Thermally Modified Grubbs $2^{\text {nd }}$ Generation Catalyst
}

\author{
Stephen Hanessian, * Simon Giroux and Andreas Larsson \\ Department of Chemistry, Université de Montréal, C.P. 6128, Succursale Centre-Ville, Montréal, Québec \\ H3C 3J7, Canada \\ stephen.hanessian@umontreal.ca
}

-Supporting Information-

Contents:

1. Generalities (S2)

2. Characterization data (S2-S12)

3. Copies of selected ${ }^{1} \mathrm{H}$ and ${ }^{13} \mathrm{C}$ NMR spectra (S13-S28)

4. HRMS spectra for deuteration experiment (S29) 


\section{Generalities:}

All commercially available starting materials (1a-4a, 11a, 16a and 22a) were used without further purification. All isomerization reaction were performed under normal atmosphere and with undistilled/undegased $\mathrm{MeOH}$. All other reactions were performed under nitrogen atmosphere. NMR $\left({ }^{1} \mathrm{H},{ }^{13} \mathrm{C}\right)$ spectra were recorded on a Bruker AV-400 spectrometer. Low- and high-resolution mass spectra were recorded on VG Micromass, AEI-MS 902 or Kratos MS-50 spectrometers using fast atom bombardement (FAB) or electrospray techniques. Optical rotations were recorded on a Perkin-Elmer 241 polarimeter in a $1 \mathrm{dm}$ cell at ambient temperature. Analytical thin-layer chromatography was performed on Merck 60F254 pre-coated silica gel plates. Visualization was performed by ultraviolet light and/or by staining with ceric ammonium molybdate or potassium permanganate. Flash column chromatography was performed using (40-60 $\mu \mathrm{m})$ silica gel at increased pressure.

\section{General procedure for isomerization of terminal olefins.}

To a solution of the olefin (1 equiv.) in $\mathrm{MeOH}(0.075 M)$ was added I (from Aldrich) (10 mol \%) at room temperature. The suspension was then heated at $60{ }^{\circ} \mathrm{C}$. After a few minutes, the insoluble catalyst (purple) dissolved completely and the resulting orangebrown solution was stirred at $60{ }^{\circ} \mathrm{C}$ until completion (typically, $3 \mathrm{~h}$ for substrates in Table 1 and $12 \mathrm{~h}$ for substrates in Table 2 and 3 ) after which time the solution was evaporated. The residue was purified by flash chromatography the give the isomerized olefin.

\section{1,2-Dimethoxy-4-propenyl-benzene (1b)}

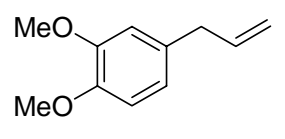

$1 a$

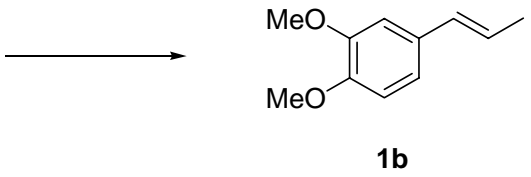

Following the general procedure with 1a, 1b was isolated in $80 \%$ yield $(E / Z, 14: 1)$. Spectral data identical to those of the literature. ${ }^{1}$

\section{2-Propenyl-phenol (2b)}




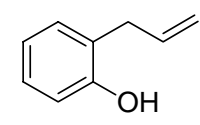

$2 a$

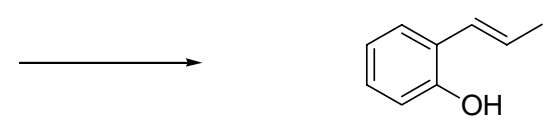

$2 b$

Following the general procedure with 2a, $2 \mathbf{b}$ was isolated in 92\% yield (E/Z, 11:1).

Spectral data identical to those of the literature. ${ }^{2}$

\section{1,2,3,4,5-Pentafluoro-6-propenyl-benzene (3b)}

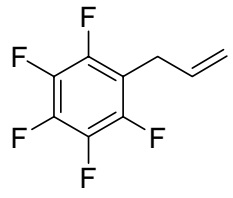

3a

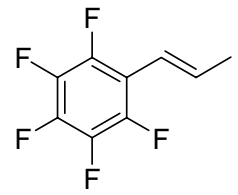

3b

Following the general procedure with 3a, $3 \mathbf{b}$ was isolated in $80 \%$ yield $(E / Z,>20: 1)$. Spectral data identical to those of the literature. ${ }^{3}$

\section{1-(3-Hydroxy-2-propenyl-phenyl)-ethanone (4b)}

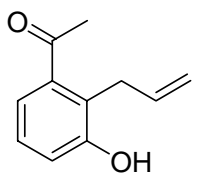

$4 a$

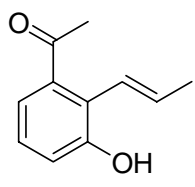

4b

Following the general procedure with $\mathbf{4 a}$, $\mathbf{4 b}$ was isolated in $89 \%$ yield $(E / Z, 11: 1)$.

${ }^{1} \mathrm{H}$ NMR (400 MHz, $\left.\mathrm{CHCl}_{3}\right) \delta(\mathrm{ppm}) 8.02(\mathrm{~s}, 1 \mathrm{H}), 7.85(\mathrm{~m}, 1 \mathrm{H}), 7.45(\mathrm{dd}, 1 \mathrm{H}, J=1.9$ and 8.4 Hz), $6.95(\mathrm{~d}, 1 \mathrm{H}, J=8.4 \mathrm{~Hz}), 6.67(\mathrm{~d}, 1 \mathrm{H}, J=16 \mathrm{~Hz}), 6.34(\mathrm{~m}, 1 \mathrm{H}), 2.61(\mathrm{~s}, 3 \mathrm{H})$, $1.93(\mathrm{~d}, 11 / 123 \mathrm{H}, J=5.4 \mathrm{~Hz}), 1.77(\mathrm{~d}, 1 / 123 \mathrm{H}, J=5.5 \mathrm{~Hz}) ;{ }^{13} \mathrm{C} \mathrm{NMR}(100 \mathrm{MHz}$, $\left.\mathrm{CDCl}_{3}\right) \delta(\mathrm{ppm}) 198.3,157.6,129.2,128.6,128.4,127.9,125.0,124.3,122.9(\mathrm{~min})$, 115.3, 25.9, 20.7(min), 18.5, 13.7(min); IR (film, $\mathrm{cm}^{-1}$ ) 3293, 1654, 1586, 1359, 1278, 1256. 


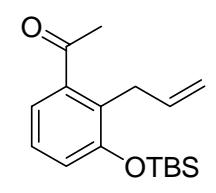

$5 a$

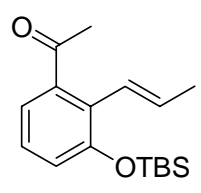

$5 b$

Following the general procedure $5 \mathbf{a}^{4}, \mathbf{5 b}$ was isolated in $90 \%$ yield $(E / Z,>15: 1)$.

${ }^{1} \mathrm{H}$ NMR $\left(400 \mathrm{MHz}, \mathrm{CHCl}_{3}\right) \delta(\mathrm{ppm}) 8.06(\mathrm{~d}, 1 \mathrm{H}, J=2.2 \mathrm{~Hz}), 7.72(\mathrm{dd}, 1 \mathrm{H}, J=2.3$ and $8.5 \mathrm{~Hz}), 6.82(\mathrm{~d}, 1 \mathrm{H}, J=8.5 \mathrm{~Hz}), 6.70(\mathrm{~d}, 1 \mathrm{H}, J=16.0 \mathrm{~Hz}), 6.27(\mathrm{~m}, 1 \mathrm{H}), 2.57(\mathrm{~s}, 3 \mathrm{H})$, $1.92\left(\mathrm{~d}, 3 \mathrm{H}_{M e, \text { major }} J=6.5 \mathrm{~Hz}\right), 1.86\left(\mathrm{~d}, 3 \mathrm{H}_{M e, \text { minor }}, J=7.1 \mathrm{~Hz}\right), 1.04(\mathrm{~s}, 9 \mathrm{H}), 0.25$ (s, $6 \mathrm{H}) ;{ }^{13} \mathrm{C} \mathrm{NMR}\left(100 \mathrm{MHz}, \mathrm{CDCl}_{3}\right) \delta(\mathrm{ppm})$ 196.8, 156.3, 130.4, 129.2, 127.9, 126.8, 126.6, 125.0, 118.7, 26.0, 25.3, 18.4, 17.9, -4.5; IR (film, $\mathrm{cm}^{-1}$ ) 2931, 2859, 1681, 1595, 1490, 1356, 1260; HRMS calcd. for $\mathrm{C}_{17} \mathrm{H}_{27} \mathrm{O}_{2} \mathrm{Si}\left(\mathrm{M}+\mathrm{H}^{+}\right)$291.1774, found: 291.1784.

\section{1-Benzenesulfonyl-2-propenyl-1H-indole (6b)}

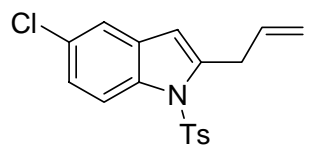

$6 \mathbf{a}$

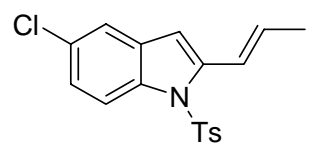

$6 b$

Following the general procedure with $\mathbf{6 a}^{5}, \mathbf{6 b}$ was isolated in $87 \%$ yield $(E / Z, 2.7: 1)$.

${ }^{1} \mathrm{H}$ NMR $\left(\mathrm{CDCl}_{3}, 400 \mathrm{MHz}\right) \delta 8.15(\mathrm{~d}, J=8.9 \mathrm{~Hz}, 1 \mathrm{H}, \mathrm{Z}$-isomer), $8.10(\mathrm{~d}, J=8.9 \mathrm{~Hz}$, 1H, E-isomer), 7.61-7.58 (m, 2H), 7.41 (d, $J=1.8 \mathrm{~Hz}, 1 \mathrm{H}, Z), 7.37$ (d, $J=1.9 \mathrm{~Hz}, 1 \mathrm{H}$, E), 7.26-7.15 (m, 3H), $7.02(\mathrm{dq}, J=20.0 \mathrm{~Hz}, 0.9 \mathrm{~Hz}, 1 \mathrm{H}, E), 6.88(\mathrm{dq}, J=11.1 \mathrm{~Hz}, 0.8$ $\mathrm{Hz}, 1 \mathrm{H}, \mathrm{Z}), 6.52$ (s, 1H; E), 6.43 (s, 1H, Z), 6.19 (dq, J=15.6 Hz, $6.8 \mathrm{~Hz}, 1 \mathrm{H}, \mathrm{E}), 6.05$ (dq, $J=11.7 \mathrm{~Hz}, 7.1 \mathrm{~Hz}, 1 \mathrm{H}, \mathrm{Z}), 2.33$ (s, 3H), 1.96 (dd, $J=6.8 \mathrm{~Hz}, 1.8 \mathrm{~Hz}, 3 \mathrm{H}, \mathrm{E}), 1.79$ $(\mathrm{dd}, J=7.1 \mathrm{~Hz}, 1.9 \mathrm{~Hz}, 3 \mathrm{H}, \mathrm{Z}) ;{ }^{13} \mathrm{C} \mathrm{NMR}\left(\mathrm{CDCl}_{3}, 100 \mathrm{MHz}\right): \delta 144.9,141.3,137.8$ (min), 135.3, 135.2, 131.6, 131.3 (min), 131.2 (min), 130.7, 129.7 (min), 129.6, 129.6, 129.4 ( $\mathrm{min}), 126.6,124.8$ ( $\mathrm{min}), 124.6$ ( $\mathrm{min}), 124.3,121.3,120.6$ ( $\mathrm{min}), 120.2$ (min), 120.0 (min), 119.8, 116.1 (min), 116.1, 111.1 (min), 107.9 (min), 106.8, 21.6, 18.8, 14.9 (min); IR (film, cm ${ }^{-1}$ ) 3080, 2956, 2923, 2851, 1597, 1445, 1372, 1170, 1090, 1047, 920, 874, 810, 716, 667; HRMS calcd for $\mathrm{C}_{18} \mathrm{H}_{16} \mathrm{ClNO}_{2} \mathrm{SAg}(\mathrm{M}+\mathrm{Ag})$ 451.9635, found 451.9655 .

\section{2-Benzyloxycarbonylamino-pent-3-enoic acid methyl ester (7b)}




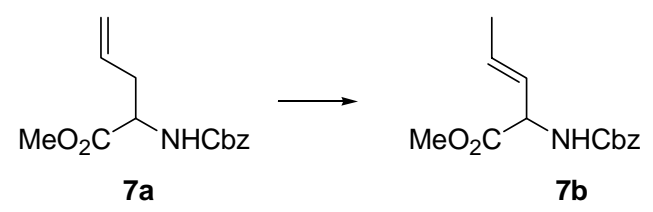

Following the general procedure with $7 \mathbf{a}^{6}, 7 \mathbf{b}$ was isolated in $76 \%$ yield $(E / Z, 4: 1)$.

${ }^{1} \mathrm{H}$ NMR $\left(400 \mathrm{MHz}, \mathrm{CHCl}_{3}\right) \delta(\mathrm{ppm}) 7.36(\mathrm{~m}, 5 \mathrm{H}), 5.81(\mathrm{~m}, 1 \mathrm{H}), 5.52(\mathrm{~m}, 1 \mathrm{H}), 5.13(\mathrm{~s}$, $2 \mathrm{H}+1 \mathrm{H}), 4.86(\mathrm{t}, 1 \mathrm{H}, J=6.9 \mathrm{~Hz}), 3.76(\mathrm{~s}, 3 \mathrm{H}), 1.84(\mathrm{~d}, 1 / 53 \mathrm{H}, J=6.5 \mathrm{~Hz}$, minor $\mathrm{Z})$, $1.73(\mathrm{~d}, 4 / 53 \mathrm{H}, J=6.5 \mathrm{~Hz}$, major $E) ;{ }^{13} \mathrm{C} \mathrm{NMR}\left(100 \mathrm{MHz}, \mathrm{CDCl}_{3}\right) \delta(\mathrm{ppm}) 171.5(\mathrm{~min})$, 171.1, 155.1, 135.8, 129.6, 128.2, 128.1, 127.9, 128.8, 127.8, 124.7, 69.3(min), 66.6, 55.3(min), 52.2, 17.3, 13.1(min); IR (film, $\mathrm{cm}^{-1}$ ) 3344, 3034, 2954, 1723, 1521, 1455, 1337, 1214, 1050; HRMS calcd. for $\mathrm{C}_{14} \mathrm{H}_{17} \mathrm{NO}_{4} \mathrm{Na}\left(\mathrm{M}+\mathrm{Na}^{+}\right)$286.1049, found: 286.1049 .

2-tert-Butoxycarbonylamino-pent-3-enoic acid methyl ester (8b)

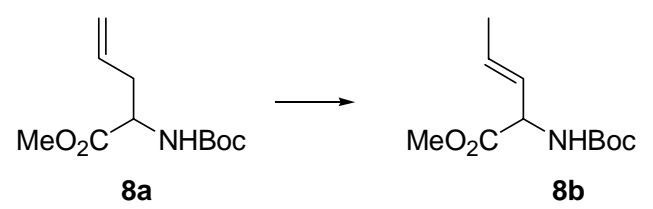

Following the general procedure with $\mathbf{8 a}^{7}, \mathbf{8 b}$ was isolated in $74 \%$ yield $(E / Z, 4: 1)$.

Spectral data identical to those of the literature. ${ }^{8}$

HRMS calcd. for for $\mathrm{C}_{11} \mathrm{H}_{19} \mathrm{NO}_{4} \mathrm{Na}\left(\mathrm{M}+\mathrm{Na}^{+}\right)$252.1206, found: 252.1194.

$(2 S, 4 R)$-2-tert-Butoxycarbonylamino-4-propenyl-pentanedioic acid dimethyl ester (9b)
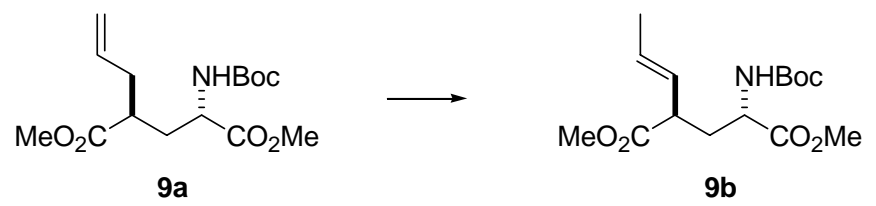

Following the general procedure with $\mathbf{9 \mathbf { a } ^ { 9 }}, \mathbf{9} \mathbf{b}$ was isolated in $96 \%$ yield (E/Z, 4:1).

$[\alpha]_{\mathrm{D}}-23.0\left(\right.$ c 0.1, $\left.\mathrm{CHCl}_{3}\right) ;{ }^{1} \mathrm{H} \mathrm{NMR}\left(400 \mathrm{MHz}, \mathrm{CHCl}_{3}\right) \delta(\mathrm{ppm}) 5.67(\mathrm{~m}, 1 \mathrm{H}), 5.38(\mathrm{~m}$, $1 \mathrm{H}), 4.93(\mathrm{~d}, 1 \mathrm{H}, J=8.5 \mathrm{~Hz}), 4.30(\mathrm{~m}, 1 \mathrm{H}), 3.72(\mathrm{~s}, 3 \mathrm{H}), 3.67$ (s, 3H), 3.10 (q, 1H, $J=$ 
$7.5 \mathrm{~Hz}$ ), $2.03(\mathrm{~m}, 2 \mathrm{H}), 1.70(\mathrm{dd}, 4 / 5$ of $3 \mathrm{H}, J=1.3$ and $6.4 \mathrm{~Hz}$ (major $E$ ) with dd, 1/5 of $3 \mathrm{H}, J=5.4 \mathrm{~Hz}$ and $6.9 \mathrm{~Hz}$ (minor Z)) $1.44(\mathrm{~m}, 1 \mathrm{H}) ;{ }^{13} \mathrm{C} \mathrm{NMR}\left(100 \mathrm{MHz}, \mathrm{CDCl}_{3}\right) \delta$ (ppm) 173.9, 172.6, 154.9, 129.7, 126.5, 126.0 (min), 79.57, 60.0, 51.9, 51.6, 51.2, 45.3, 39.8, 34.2, 34.0 (min), 27.8, 20.6 ( $\mathrm{min}), 17.6,13.8$ (min) 213.5, 128.7, 127.4, 59.6, 34.5(2), 17.8, 17.5; IR (film, cm ${ }^{-1}$ ) 3368, 2978, 1737, 1717, 1521, 1438, 1367, 1163; LRMS (ES+) $316.8\left(\mathrm{M}+\mathrm{H}^{+}, 25\right), 216.5(\mathrm{M}-\mathrm{Boc}, 100)$; HRMS calcd. for $\mathrm{C}_{15} \mathrm{H}_{25} \mathrm{NO}_{6} \mathrm{Na}$ $\left(\mathrm{M}+\mathrm{Na}^{+}\right)$338.1574, found: 338.1572 .

1-Methyl-3-propenyl-pyrrolidin-2-one (10b)

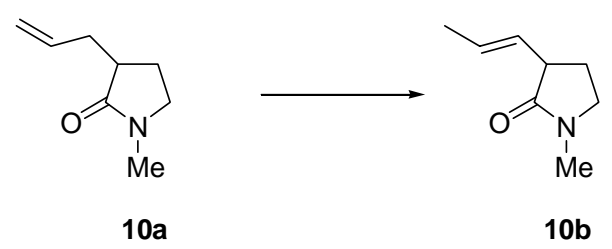

Following the general procedure with $10 \mathbf{a}^{10}, \mathbf{1 0 b}$ was isolated in $80 \%$ yield $(E / Z, 4: 1)$.

${ }^{1} \mathrm{H}$ NMR (400 MHz, $\left.\mathrm{CHCl}_{3}\right) \delta(\mathrm{ppm}) 5.60(\mathrm{~m}, 1 \mathrm{H}), 5.48(\mathrm{~m}, 1 \mathrm{H}), 3.30(\mathrm{~m}, 2 \mathrm{H}), 3.02$ (q, $1 \mathrm{H}, J=8.2 \mathrm{~Hz}), 2.83(\mathrm{~s}, 3 \mathrm{H}), 2.28(\mathrm{~m}, 1 \mathrm{H}), 1.86(\mathrm{~m}, 1 \mathrm{H}) ;{ }^{13} \mathrm{C} \mathrm{NMR}\left(100 \mathrm{MHz}, \mathrm{CDCl}_{3}\right) \delta$ (ppm) 174.9, 127.9, 127.7, 127.6(min), 127.4(min), 47.3(min), 47.1, 44.8, 40.0(min), 29.6(min), 29.5, 25.7(min), 25.0, 17.6, 12.9(min); IR (film, $\mathrm{cm}^{-1}$ ) 2941, 2881, 1678, 1500, 1438, 1404, 1300; HRMS calcd. for $\mathrm{C}_{8} \mathrm{H}_{14} \mathrm{NO}\left(\mathrm{M}+\mathrm{H}^{+}\right)$140.1069, found: 140.1073.

\section{2-Propenyl-cyclohexanone (11b)}

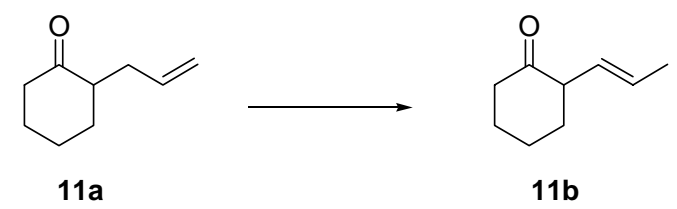

Following the general procedure with 11a, 11b was isolated in $90 \%$ yield $(E / Z, 5: 1)$. Spectral data identical to those of the literature. ${ }^{11}$

6-(tert-Butyl-diphenyl-silanyloxy)-2-propenyl-hexanoic acid ethyl ester (12b) 


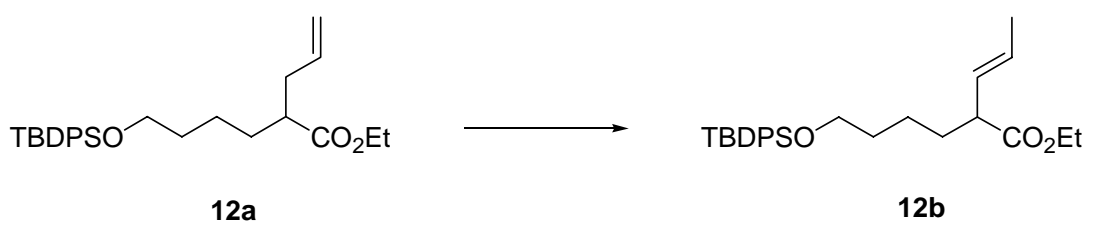

Following the general procedure with $\mathbf{1 2 a}^{12}, \mathbf{1 2} \mathbf{b}$ was isolated in $95 \%$ yield $(E / Z, 4: 1)$.

${ }^{1} \mathrm{H}$ NMR $\left(400 \mathrm{MHz}, \mathrm{CHCl}_{3}\right) \delta(\mathrm{ppm}) 7.71(\mathrm{~m}, 4 \mathrm{H}), 7.43(\mathrm{~m}, 6 \mathrm{H}), 5.62(\mathrm{~m}, 1 \mathrm{H}), 5.45(\mathrm{~m}$, 1H), 4.16 (q, 2H, $J=7.1 \mathrm{~Hz}), 3.69$ (t, $2 \mathrm{H}, J=6.3 \mathrm{~Hz}$ ), 3.35 (m, 1/5H), 2.96 (q, 4/5H, J = $7.6 \mathrm{~Hz}), 1.71(\mathrm{~d}, 3 \mathrm{H}$ from Me, $J=6.3 \mathrm{~Hz}+1 \mathrm{H}), 1.65-1.33(\mathrm{~m}, 5 \mathrm{H}), 1.28(\mathrm{t}, 3 \mathrm{H}, J=7.1$ $\mathrm{Hz}), 1.09$ (s, 9H); ${ }^{13} \mathrm{C} \mathrm{NMR}\left(100 \mathrm{MHz}, \mathrm{CDCl}_{3}\right) \delta$ (ppm) 174.3, 174.2(min), 135.2, 133.6, 129.1, 128.5, 128.1, 127.5, 127.2, 126.2, 63.3, 59.9, 49.0, 43,4(min), 32.1(min), 31.9(2), 26.4, 23.0, 18.8, 17.5, 13.9, 12.8(min); IR (film, $\mathrm{cm}^{-1}$ ) 2933, 2859, 1734, 1428, 1111; HRMS calcd. for $\mathrm{C}_{27} \mathrm{H}_{39} \mathrm{O}_{3} \mathrm{Si}\left(\mathrm{M}+\mathrm{H}^{+}\right) 439.2663$, found: 439.2672 .

\section{Propenyl-santonin (13b)}

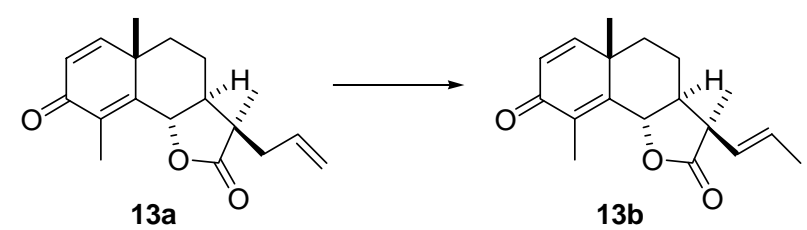

Following the general procedure in the absence of light with $\mathbf{1 3 \mathbf { a } ^ { 1 3 }}, \mathbf{1 3 \mathbf { b }}$ was isolated in $90 \%$ yield $(E / Z,>20: 1)$.

$[\alpha]_{\mathrm{D}}-208.0\left(\right.$ c $\left.0.1, \mathrm{CHCl}_{3}\right)$; m.p. $119-120{ }^{\circ} \mathrm{C} ;{ }^{1} \mathrm{H}$ NMR $\left(400 \mathrm{MHz}, \mathrm{C}_{6} \mathrm{D}_{6}\right) \delta$ (ppm) 6.35 $(\mathrm{d}, 1 \mathrm{H}, \mathrm{J}=9.8 \mathrm{~Hz}), 6.09$, (d, 1H, $\mathrm{J}=9.8 \mathrm{~Hz}), 5.62(\mathrm{qd}, 1 \mathrm{H}, \mathrm{J}=6.5$ and $15.8 \mathrm{~Hz}), 5.12$ $(\mathrm{dd}, 1 \mathrm{H}, \mathrm{J}=1.5$ and $15.8 \mathrm{~Hz}), 4.57(\mathrm{dd}, 1 \mathrm{H}, \mathrm{J}=1.1$ and $11.6 \mathrm{~Hz}), 2.61(\mathrm{~s}, 3 \mathrm{H}), 1.57(\mathrm{~d}$, $3 \mathrm{H}, \mathrm{J}=6.4 \mathrm{~Hz}), 1.49(\mathrm{~m}, 1 \mathrm{H}), 1.23(\mathrm{~s}, 3 \mathrm{H}), 1.15(\mathrm{~m}, 2 \mathrm{H}), 0.98(\mathrm{~m}, 2 \mathrm{H}), 0.74(\mathrm{~s}, 3 \mathrm{H}) ;{ }^{13} \mathrm{C}$ NMR (100 MHz, $\left.\mathrm{C}_{6} \mathrm{D}_{6}\right) \delta(\mathrm{ppm})$ 184.7, 176.4, 153.4, 150.4, 128.5, 128.0, 125.9, 125.5, 78.2, 55.8, 48.2, 40.2, 36.7, 24.0, 21.0, 18.2, 17.4, 10.8; IR (film, $\mathrm{cm}^{-1}$ ) 2968, 2934, 1782, 1663, 1636, 1615, 1038; HRMS calcd. for $\mathrm{C}_{18} \mathrm{H}_{23} \mathrm{O}_{3}\left(\mathrm{M}+\mathrm{H}^{+}\right)$287.1641, found: 287.1634 .

(2S, 3S, 5S)-(2-Benzyloxymethoxy-3,5-dimethyl-oct-6-enyloxy)-tert-butyl-diphenylsilane (14b) 


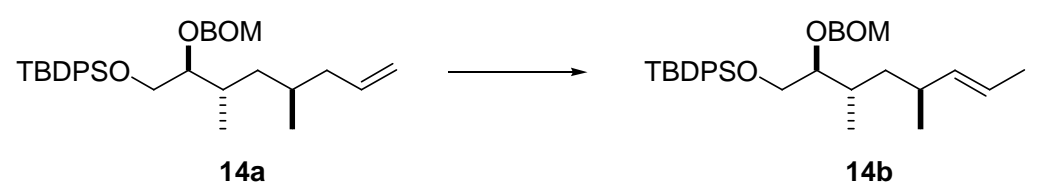

Following the general procedure with $\mathbf{1 4} \mathbf{a}^{14}, \mathbf{1 4} \mathbf{b}$ was isolated in $90 \%$ yield $(E / Z, 5: 1)$.

$[\alpha]_{\mathrm{D}}-19.7\left(c 1.0, \mathrm{CHCl}_{3}\right) ;{ }^{1} \mathrm{H} \mathrm{NMR}\left(400 \mathrm{MHz}, \mathrm{CHCl}_{3}\right) \delta(\mathrm{ppm}) 7.74(\mathrm{~m}, 4 \mathrm{H}), 7.36(\mathrm{~m}$, $11 \mathrm{H}), 5.39(\mathrm{~m}, 2 \mathrm{H}), 4.97(\mathrm{~d}, 1 \mathrm{H}, J=6.9 \mathrm{~Hz}), 4.87(\mathrm{~d}, 1 \mathrm{H}, J=6.9 \mathrm{~Hz}), 4.72(\mathrm{~d}, 1 \mathrm{H}, J=$ $11.7 \mathrm{~Hz}), 4.62(\mathrm{~d}, 1 \mathrm{H}, J=11.7 \mathrm{~Hz}), 3.76(\mathrm{~m}, 3 \mathrm{H}), 2.09(\mathrm{~m}, 1 \mathrm{H}), 1.98(\mathrm{~m}, 1 \mathrm{H}), 1.66(\mathrm{~d}$, $3 \mathrm{H}, J=6.5 \mathrm{~Hz}$ with minor $\mathrm{Z}), 1.32(\mathrm{~m}, 1 \mathrm{H}), 1.10(\mathrm{~s}, 9 \mathrm{H}+1 \mathrm{H}), 0.93(\mathrm{~m} 8 \mathrm{H}$ with $\mathrm{d}$ of $6 \mathrm{H}$ $J=6.7 \mathrm{~Hz}) ;{ }^{13} \mathrm{C} \mathrm{NMR}\left(100 \mathrm{MHz}, \mathrm{CDCl}_{3}\right) \delta(\mathrm{ppm}) 137.7,137.5,137.1(\mathrm{~min}), 136.4(\mathrm{~min})$, 135.3, 135.2, 133.1, 129.3, 129.2, 128.0, 127.4, 127.3, 127.2, 127.1, 123.1(min), 122.2, 121.5(min), 94.5(min), 94.3, 82.4(min), 81.6, 69.2(min), 69.1, 63.8, 39.4(min), 39.2, 33.8, 32.2( $\mathrm{min}), 32.0,31.8(\mathrm{~min}), 28.5(\mathrm{~min}), 26.4,20.0(\mathrm{~min}), 19.8,18.8,17.6,15.4(\mathrm{~min})$, 15.3, 15.0(min); IR (film, cm ${ }^{-1}$ ) 3071, 2959, 1455, 1428, 1379, 1112, 1041; LRMS (ES+) $531.3\left(\mathrm{M}+\mathrm{H}^{+}, 25\right)$; HRMS calcd. for $\mathrm{C}_{34} \mathrm{H}_{46} \mathrm{O}_{3} \mathrm{SiNa}\left(\mathrm{M}+\mathrm{Na}^{+}\right)$553.3108, found: 553.3113 .

\section{2-Methyl-2-propenyl-cyclopentane-1,3-dione (15b)}

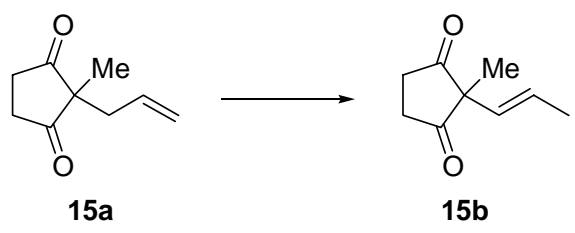

Following the general procedure with 15a, 15b was isolated in $92 \%$ yield $(E / Z,>20: 1)$.

${ }^{1} \mathrm{H}$ NMR $\left(400 \mathrm{MHz}, \mathrm{CHCl}_{3}\right) \delta(\mathrm{ppm}) 5.54(\mathrm{~m}, 1 \mathrm{H}), 5.32(\mathrm{dd}, 1 \mathrm{H}, J=1.5$ and $15.6 \mathrm{~Hz})$, 3.00-2.58 (m, 4H), 1.67 (dd, 3H, $J=1,5$ and $6.5 \mathrm{~Hz}), 1.19$ (s, 3H); ${ }^{13} \mathrm{C}$ NMR $(100 \mathrm{MHz}$, $\left.\mathrm{CDCl}_{3}\right) \delta$ (ppm) 213.5, 128.7, 127.4, 59.6, 34.5(2), 17.8, 17.5; IR (film, $\mathrm{cm}^{-1}$ ) 2933, 1767, 1725, 1450, 1421, 1256, 1031; HRMS calcd. for $\mathrm{C}_{9} \mathrm{H}_{13} \mathrm{O}_{2}\left(\mathrm{M}+\mathrm{H}^{+}\right)$153.0910, found: 153.0911 .

\section{4-[1-(tert-Butyl-dimethyl-silanyloxy)-but-2-enyl]-quinoline (16b)}




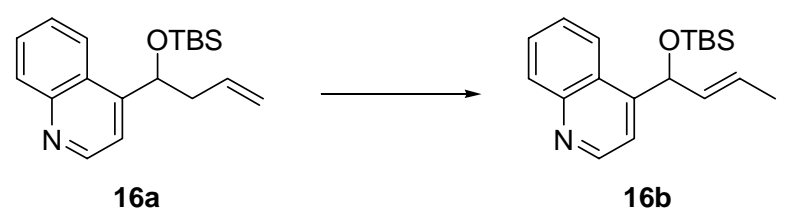

Following the general procedure with $\mathbf{1 6 a}^{15}, \mathbf{1 6 b}$ was isolated in $74 \%$ yield $(E / Z, 10: 1)$.

${ }^{1} \mathrm{H}$ NMR (400 MHz, $\left.\mathrm{CHCl}_{3}\right) \delta(\mathrm{ppm}) 8.92(\mathrm{~d}, 1 \mathrm{H}, J=4.5 \mathrm{~Hz}), 8.13(\mathrm{~d}, 1 \mathrm{H}, J=8.1 \mathrm{~Hz})$, $8.10(\mathrm{~d}, 1 \mathrm{H}, J=8.5 \mathrm{~Hz}), 7.71$ (t, 1H, $J=8.3 \mathrm{~Hz}), 7.60$ (d, 1H, $J=4.5 \mathrm{~Hz}), 7.55(\mathrm{~d}, 1 \mathrm{H}, J$ $=7.0 \mathrm{~Hz}), 5.80-5.60(\mathrm{~m}, 3 \mathrm{H}), 1.95\left(\mathrm{~d}, 1 / 113 \mathrm{H}_{\text {minor,Me }}, J=5.4 \mathrm{~Hz}\right), 1.66(\mathrm{~d}, 9 / 11$ $\left.3 \mathrm{H}_{\text {major,Me }}, J=6.2 \mathrm{~Hz}\right), 0.94(\mathrm{~s}, 9 \mathrm{H}), 0.12(\mathrm{~s}, 3 \mathrm{H}), 0.02(\mathrm{~s}, 3 \mathrm{H}) ;{ }^{13} \mathrm{C}$ NMR $(100 \mathrm{MHz}$, $\left.\mathrm{CDCl}_{3}\right) \delta(\mathrm{ppm}) 150.1,149.2,147.9,132.5,129.8,128.4,126.6,125.7,125.0,123.4$, 117.5, 71.9, 25.4, 17.9, 17.3, -4.9, -5.2; IR (film, $\mathrm{cm}^{-1}$ ) 2955, 2929, 1591, 1508, 1471, 1253, 1092; HRMS calcd. for $\mathrm{C}_{19} \mathrm{H}_{28} \mathrm{NOSi}\left(\mathrm{M}+\mathrm{H}^{+}\right) 314.1934$, found: 314.1940 .

\section{Acetic acid 1-quinolin-4-yl-but-2-enyl ester (17b)}

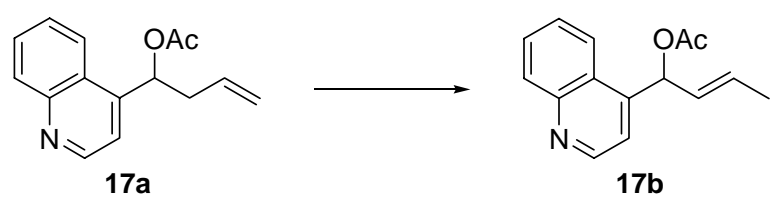

Following the general procedure with $\mathbf{1 7} \mathbf{a}^{15}, \mathbf{1 7} \mathbf{b}$ was isolated in $61 \%$ yield $(E / Z, 5: 1)$.

${ }^{1} \mathrm{H}$ NMR $\left(400 \mathrm{MHz}, \mathrm{CHCl}_{3}\right) \delta(\mathrm{ppm}) 8.92(\mathrm{~d}, 1 \mathrm{H}, J=4.3 \mathrm{~Hz}), 8.15(\mathrm{~d}, 1 \mathrm{H}, J=8.4 \mathrm{~Hz})$, 8.08 (d, 1H, $J=8.4 \mathrm{~Hz}$ ), 7.73 (t, 1H, $J=7.0 \mathrm{~Hz}), 7.58$ (m, 1H), 7.52-7.44 (m, 1H), 7.22 $(\mathrm{d}, 1 / 6 \mathrm{H}, J=8.9 \mathrm{~Hz}), 6.90(\mathrm{~d}, 5 / 6 \mathrm{H}, J=5.4 \mathrm{~Hz}), 5.82(\mathrm{~m}, 2 \mathrm{H}), 2.17$ (s, 3H), $1.94(\mathrm{~d}, 1 / 6$ of $3 \mathrm{H}, J=6.9 \mathrm{~Hz}), 1.71$ (d, $5 / 6$ of $3 \mathrm{H}, J=5.2 \mathrm{~Hz}) ;{ }^{13} \mathrm{C} \mathrm{NMR}\left(100 \mathrm{MHz}, \mathrm{CDCl}_{3}\right) \delta(\mathrm{ppm})$ 169.4, 149.8, 148.0, 144.4, 131.2, 129.9, 128.8, 127.6, 126.3, 125.0, 123.2, 118.0, 72.0, 20.8, 17.4. IR (film, $\mathrm{cm}^{-1}$ ) 2935, 1743, 1593, 1508, 1231, 1036; HRMS calcd. for $\mathrm{C}_{15} \mathrm{H}_{16} \mathrm{NO}_{2}\left(\mathrm{M}+\mathrm{H}^{+}\right) 242.1175$, found: 242.1176

\section{Acetic acid 1-(4-nitro-phenyl)-but-2-enyl ester (18b)}

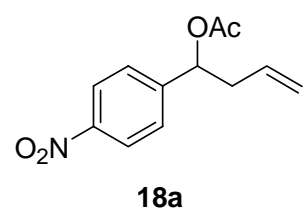

$18 \mathbf{a}$
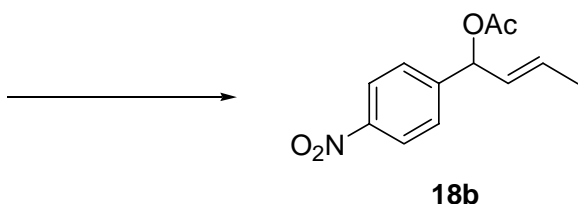
Following the general procedure with $\mathbf{1 8} \mathbf{a}^{16}, \mathbf{1 8 b}$ was isolated in $93 \%$ yield $(E / Z, 7: 1)$.

${ }^{1} \mathrm{H}$ NMR (400 MHz, $\left.\mathrm{CHCl}_{3}\right) \delta(\mathrm{ppm}) 8.21(\mathrm{~d}, 1 \mathrm{H}, J=8.8 \mathrm{~Hz}), 7.50(\mathrm{~d}, 1 \mathrm{H}, J=8.6 \mathrm{~Hz})$, $6.65(\mathrm{~d}, 1 / 8 \mathrm{H}, J=9.1 \mathrm{~Hz}), 6.26(\mathrm{~d}, 7 / 8 \mathrm{H}, J=7.2 \mathrm{~Hz}), 5.80(\mathrm{~m}, 1 \mathrm{H}), 5.60(\mathrm{~m}, 1 \mathrm{H}), 2.14(\mathrm{~s}$, $3 \mathrm{H}), 1.85(\mathrm{~d}, 1 / 8$ of $3 \mathrm{H}, J=7.0 \mathrm{~Hz}), 1.74(\mathrm{~d}, 7 / 8$ of $3 \mathrm{H}, J=6.6 \mathrm{~Hz}) ;{ }^{13} \mathrm{C}$ NMR $(100 \mathrm{MHz}$, $\left.\mathrm{CDCl}_{3}\right) \delta(\mathrm{ppm}) 169.4,146.6,130.9,127.9,127.0,123.4,74.9,20.8,17.4$; IR (film, $\mathrm{cm}^{-1}$ ) 2944, 1736, 1606, 1521, 1348, 1228; HRMS calcd. for $\mathrm{C}_{12} \mathrm{H}_{13} \mathrm{O}_{4} \mathrm{Na}\left(\mathrm{M}+\mathrm{Na}^{+}\right)$258.0736, found: 258.0782 .

tert-Butyl-dimethyl-(1-phenyl-but-2-enyloxy)-silane (19b)

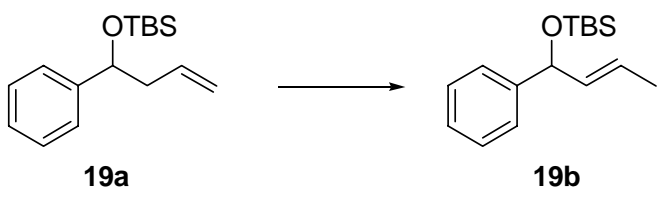

Following the general procedure with $19 \mathbf{a}^{17}, \mathbf{1 9 b}$ was isolated in $92 \%$ yield $(E / Z, 9: 1)$.

${ }^{1} \mathrm{H}$ NMR (400 MHz, $\left.\mathrm{CHCl}_{3}\right) \delta(\mathrm{ppm}) 7.35(\mathrm{~m}, 5 \mathrm{H}), 5.65(\mathrm{~m}, 1 \mathrm{H}), 5.60(\mathrm{~m}, 1 \mathrm{H}), 5.15($, d, $1 \mathrm{H}, \mathrm{J}=6.4 \mathrm{~Hz}), 1.83(\mathrm{~d}, 1 / 10$ of $3 \mathrm{H}, \mathrm{J}=5.4 \mathrm{~Hz}), 1.71(\mathrm{~d}, 9 / 10$ of $3 \mathrm{H}, \mathrm{J}=6.1 \mathrm{~Hz}), 0.94(\mathrm{~s}$, 9H), 0.09 (s, 3H), 0.03 (s, 3H); ); $\left.{ }^{13} \mathrm{C} \mathrm{NMR} \mathrm{(100} \mathrm{MHz,} \mathrm{CDCl}_{3}\right) \delta(\mathrm{ppm})$ 144.1, 134.6, 127.7, 126.4, 125.4, 125.1, 124.7, 75.2, 69.4(min), 25.5, 17.9, 17.2, -4.8, -5.1; IR (film, $\mathrm{cm}^{-1}$ ) 2930, 2857, 1692, 1451, 1230, 1112; LRMS (ES+) 249.1 (M - Me $\mathrm{TBS}_{\mathrm{TBS}}$ 20), 233.1 (M- $\left.2 \mathrm{Me}_{\mathrm{TBS}}, 85\right)$.

tert-Butyl-[1-(4-chloro-phenyl)-but-2-enyloxy]-dimethyl-silane (20b)

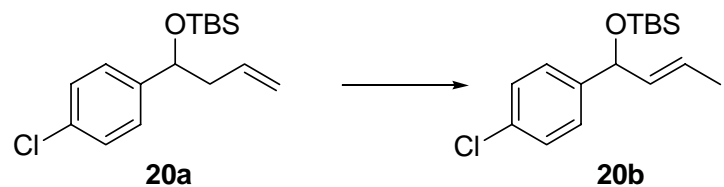

Following the general procedure with $20 \mathbf{a}^{17}$, 20b was isolated in $85 \%$ yield $(E / Z, 9: 1)$. ${ }^{1} \mathrm{H}$ NMR $\left(400 \mathrm{MHz}, \mathrm{CHCl}_{3}\right) \delta(\mathrm{ppm}) 7.30(\mathrm{~m}, 4 \mathrm{H}), 5.69(\mathrm{~m}, 1 \mathrm{H}), 5.55(\mathrm{dd}, 1 \mathrm{H}, J=6.5$ and $14.4 \mathrm{~Hz}), 5.13(\mathrm{~d}, 1 \mathrm{H}, J=6.5 \mathrm{~Hz}), 1.82(\mathrm{~d}, 1 / 10$ of $3 \mathrm{H}, J=5.4 \mathrm{~Hz}), 1.72(\mathrm{~d}, 9 / 10$ of $3 \mathrm{H}, J=6.4 \mathrm{~Hz}), 0.95(\mathrm{~s}, 9 \mathrm{H}), 0.10(\mathrm{~s}, 3 \mathrm{H}), 0.05(\mathrm{~s}, 3 \mathrm{H}) ;{ }^{13} \mathrm{C} \mathrm{NMR}\left(100 \mathrm{MHz}, \mathrm{CDCl}_{3}\right) \delta$ (ppm) 142.7, 134.3, 134.0(min), 132.0, 127.8, 126.9, 126.5 (min), 125.1, 123.1(min), 
116.8(min), 74.6, 68.9(min), 25.5, 25.4(min), 17.9, 17.1, -4.8, -5.1; IR (film, $\left.\mathrm{cm}^{-1}\right)$ 2956, 2929, 2857, 1488, 1253, 1087; HRMS calcd. for $\mathrm{C}_{16} \mathrm{H}_{25} \mathrm{OSiClAg}(\mathrm{M}+\mathrm{Ag})$ 403.0435, found: 403.0420 .

\section{1-Propenyldiphenylphosphine oxide (21b)}

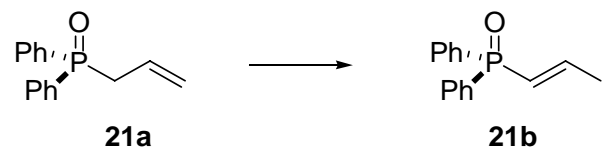

Following the general procedure with 21a, 21b was isolated in $85 \%$ yield $(E / Z,>20: 1)$. Spectral data identical to those of the literature. ${ }^{18}$

HRMS calcd. for $\mathrm{C}_{15} \mathrm{H}_{15} \mathrm{OPNa}\left(\mathrm{M}+\mathrm{Na}^{+}\right)$265.0752, found: 265.0760.

$(1 R, 2 R, 3 S, 4 R, 5 R)-5-(2,2-D i m e t h y l-[1,3]$ dioxolan-4-yl)-2,2-dimethyl-6propenyloxy-tetrahydro-furo[2,3-d][1,3] dioxole (22b)
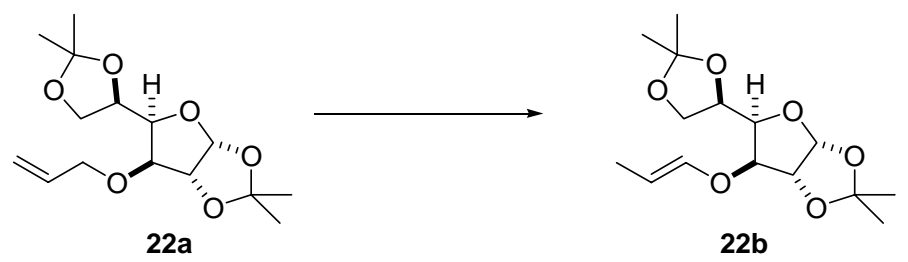

Following the general procedure with $22 \mathbf{a}^{29}, \mathbf{2 2 b}$ was isolated in $75 \%$ yield $(E / Z, 2: 1)$. $[\alpha]_{\mathrm{D}}-9.8\left(c\right.$ 0.26, $\left.\mathrm{CHCl}_{3}\right) ;{ }^{1} \mathrm{H} \mathrm{NMR}\left(400 \mathrm{MHz}, \mathrm{CHCl}_{3}\right) \delta(\mathrm{ppm}) 6.16(\mathrm{dd}, 1 / 3 \mathrm{H}, J=1.6$ and $12.5 \mathrm{~Hz}), 6.04(\mathrm{dd}, 2 / 3 \mathrm{H}, J=1.6$ and $6.1 \mathrm{~Hz}), 5.92(\mathrm{~d}, 2 / 3 \mathrm{H}, J=3.7 \mathrm{~Hz}), 5.87$ (d, $1 / 3 \mathrm{H}, J=3.7 \mathrm{~Hz}), 4.95(\mathrm{sx}, 1 / 3 \mathrm{H}, J=3.8 \mathrm{~Hz}), 4.57-4.48(\mathrm{~m}, 1 \mathrm{H}+2 / 3 \mathrm{H}), 4.32$ (qu, $1 \mathrm{H}$, $J=7.2 \mathrm{~Hz}), 4.23(\mathrm{~d}, 1 / 3 \mathrm{H}, \mathrm{J}=2.9 \mathrm{~Hz}), 4.19-4.15(\mathrm{~m}, 1 \mathrm{H}+2 / 3 \mathrm{H}), 4.12-4.00(\mathrm{~m}, 2 \mathrm{H})$, $1.56(\mathrm{~m}, 3 \mathrm{H}), 1.51(\mathrm{~s}, 3 \mathrm{H}), 1.43(\mathrm{~s}, 3 \mathrm{H}), 1.34(\mathrm{~s}, 3 \mathrm{H}), 1.31(\mathrm{~s}, 3 \mathrm{H}) ;{ }^{13} \mathrm{C} \mathrm{NMR}(100 \mathrm{MHz}$, $\left.\mathrm{CDCl}_{3}\right) \delta(\mathrm{ppm}) 144.2,143.5,111.6,111.4,108.7,108.6,104.7(2), 102.9,101.3,82.6$, $82.4,81.7,80.5,80.3,80.0,71.9,71.7,66.6(2), 26.4(2), 26.3,25.8,24.9,12.0,8.8$; IR (film, $\mathrm{cm}^{-1}$ ) 2928, 2936, 1670, 1373, 1255, 1216, 1165, 1131, 1076; HRMS calcd. for $\mathrm{C}_{15} \mathrm{H}_{25} \mathrm{O}_{6}\left(\mathrm{M}+\mathrm{H}^{+}\right)$301.1645, found: 301.1647 .

\section{5-Chloro-1-propenyl-1H-indole-3-carbaldehyde (23b)}



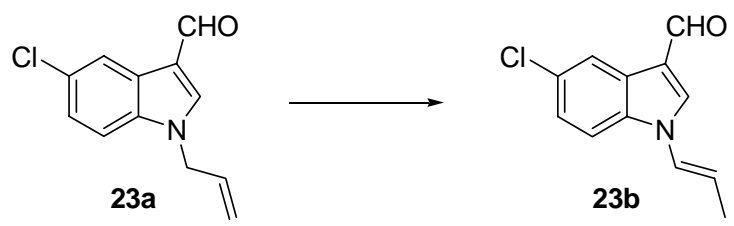

Following the general procedure with $23 \mathbf{a}^{20}, \mathbf{2 3 b}$ was isolated in $69 \%$ yield $(E / Z, 4: 1)$.

${ }^{1} \mathrm{H}$ NMR (400 MHz, $\mathrm{CHCl}_{3}$ ) $\delta(\mathrm{ppm}) 10.03$ (s, 1/5H), 9.97 (s, 4/5H), 7.85 (s, 4/5H), 7.76

(s, 1/5H), 7.34-7.20 (m, 2H), 6.87 (d, 4/5H, $J=13.9 \mathrm{~Hz}), 6.76(\mathrm{~d}, 1 / 5 \mathrm{H}, J=8.3 \mathrm{~Hz}), 6.02$

$(\mathrm{dq}, 4 / 5 \mathrm{H}, J=6.8 \mathrm{~Hz}$ and $13.8 \mathrm{~Hz}) ; 5.89(\mathrm{~m}, 1 / 5 \mathrm{H}), 1.95(\mathrm{~m}, 4 / 5 \mathrm{H}), 1.95$ (d, 4/5 of 3H, $J$ $=6.8 \mathrm{~Hz}), 1.80(\mathrm{~d}, 1.5$ of $3 \mathrm{H}, J=7.0 \mathrm{~Hz}) ;{ }^{13} \mathrm{C} \mathrm{NMR}\left(100 \mathrm{MHz}, \mathrm{CDCl}_{3}\right) \delta(\mathrm{ppm}) 184.1$, 138.2(min), 135.2, 134.4, 128.8, 125.6, 124.3, 124.2(min), 123.3, 122.9, 121.3, 121.2(min), 118.2(min), 117.9, 111.4(min), 110.8, 14.9, 12.5(min); IR (film, cm $\left.{ }^{-1}\right)$ 3110, 2791, 1663, 1530, 1455, 1404, 1180; HRMS calcd. for $\mathrm{C}_{12} \mathrm{H}_{11} \mathrm{CINO}\left(\mathrm{M}+\mathrm{H}^{+}\right) 220.0523$, found: 220.0525 .

\section{References:}

1) Robert, J. C.; Pincock, J. A. J. Org. Chem. 2006, 71, 1480-1492.

2) van Oterllo, W. A. L.; Ngidi, A. L.; Kuzvidza, S.; Morgans, G. L.; Moleele, S. S.; de Koning, C. B. Tetrahedron 2005, 61, 9996-10006.

3) Fields, R.; Mowthorpe, D. J. J. Chem. Soc. (B) 1968, 308-312.

4) 5 a was prepared via silylation ( $\mathrm{TBSCl}, \mathrm{NEt}_{3}, \mathrm{CH}_{2} \mathrm{Cl}_{2}$ ) of compound $\mathbf{4 a}$.

5) 6a was prepared via 3 steps (1. TsCl, $\mathrm{NaOH}, \mathrm{TBAHSO}_{4}, \mathrm{CH}_{2} \mathrm{Cl}_{2}$ 2. $t$ - $\mathrm{BuLi}, \mathrm{I}_{2}, \mathrm{Et}_{2} \mathrm{O},-78{ }^{\circ} \mathrm{C}, 3 . \mathrm{iPrMgBr}$, $\mathrm{CuCN} 2 \mathrm{LiCl}$, allylBr, THF, $-30{ }^{\circ} \mathrm{C}$ ) from commercially available 5-chloroindole.

6) Abbott, S. D.; Lane-Bell, P.; Sidhu, K. P. S.; Vederas, J. C. J. Am. Chem. Soc. 1994, 116, 6513-6520.

7) De Nicola, A.; Einhorn, J.; Luche, J.-L. Tetrahedron Lett. 1992, 33, 6461-6464.

8) Ichikawa, Y.; Tsuboi, K.; Isobe, M. J. Chem. Soc.; Perkin Trans. 1. 1994, 2791-2796.

9) For the preparation of 9a see: Hanessian, S.; Margarita, R. Tetrahedron Lett. 1998, 39, 5887-5890.

10) 10a was prepared via allylation (KHMDS,allylBr, THF, $-78{ }^{\circ} \mathrm{C}$ ) of commercially available $N$ methylpyrrolidinone.

11) Brown, M. J.; Harrison, T.; Herrinton, P. M.; Hopkins, M. H.; Hutchinson, K. D.; Mishra, P.; Overman, L. E. J. Am. Chem. Soc. 1991, 113, 5365-5378.

12) 12a was prepared by $O$-protection and $C$-allylation (1. TBDPSCl, $\mathrm{NEt}_{3} ; 2$. KHMDS, allylBr, THF, -78

${ }^{\circ} \mathrm{C}$ ) of commercially available Ethyl 6-hydroxyhexanoate.

13)Adekenov, S. M.; Gafurov, N. M. Khim. Prir. Soedin. 1992, 5, 518-522.

14) 15a was prepared via two steps (1. oxidation, 2. Wittig olefination) from the corresponding primary alcohol, see: Hanessian, S.; Yang, Y.; Giroux, S.; Mascitti, V.; Ma, J.; Raeppel, F. J. Am. Chem. Soc. 2003, 125, 13784-13792.

15) 16a and 17a were prepared via two steps (1. allylMgBr, $\mathrm{Et}_{2} \mathrm{O},-40{ }^{\circ} \mathrm{C}, 2$. $\mathrm{TBSCl}, \mathrm{NEt}_{3}$ for 16a and $\mathrm{Ac}_{2} \mathrm{O}, \mathrm{NEt}_{3}$, DMAP for 17a) from commercially available 4-quinoline carboxaldehyde.

16) Yadav, J. S.; Subba Reddy, B. V.; Kiran Kumar Reddy, G. S. Tetrahedron Lett, 2000, 41, 2695-2697.

17) 19a and 20a were prepared by silylation ( $\mathrm{TBSCl}, \mathrm{NEt}_{3}$ ) of commercially available 4-Phenyl-1-butenol and 1-(4-Chlorophenyl)-3-buten-1-ol respectively.

18) Demchuk, O. M.; Pietrusiewicz, K. M.; Michrowska, A.; Grela, K. Org. Lett. 2003, 5, 3217-3220

19) Murakami, H.; Minami, T.; Ozawa, F. J. Org. Chem. 2004, 69, 4482-4486.

20) 23a was prepared via allylation (allylBr, $\mathrm{K}_{2} \mathrm{CO}_{3}, \mathrm{CH}_{3} \mathrm{CN}$ ) of commercially available 5-chloroindole-3carboxaldehyde. 

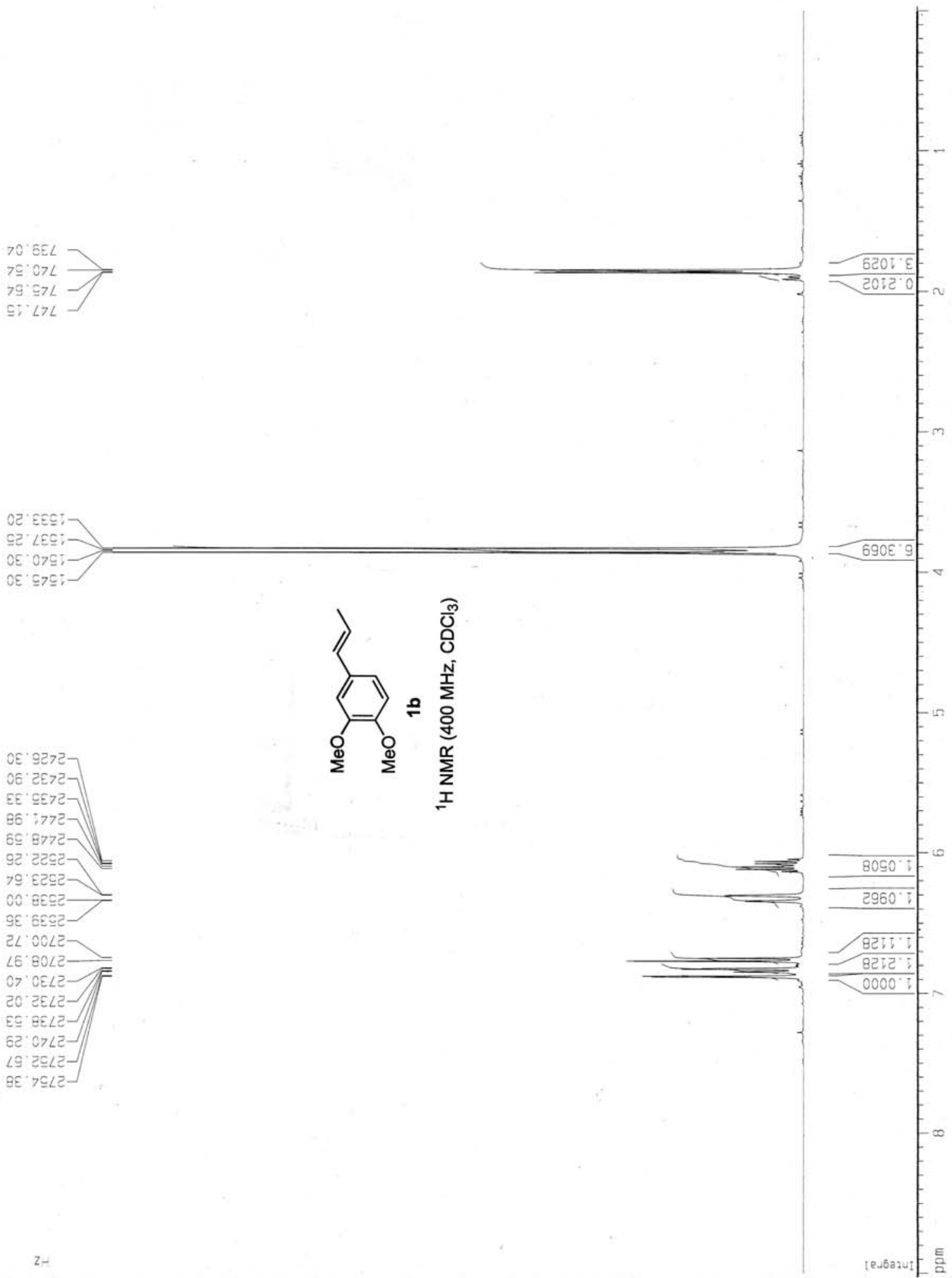


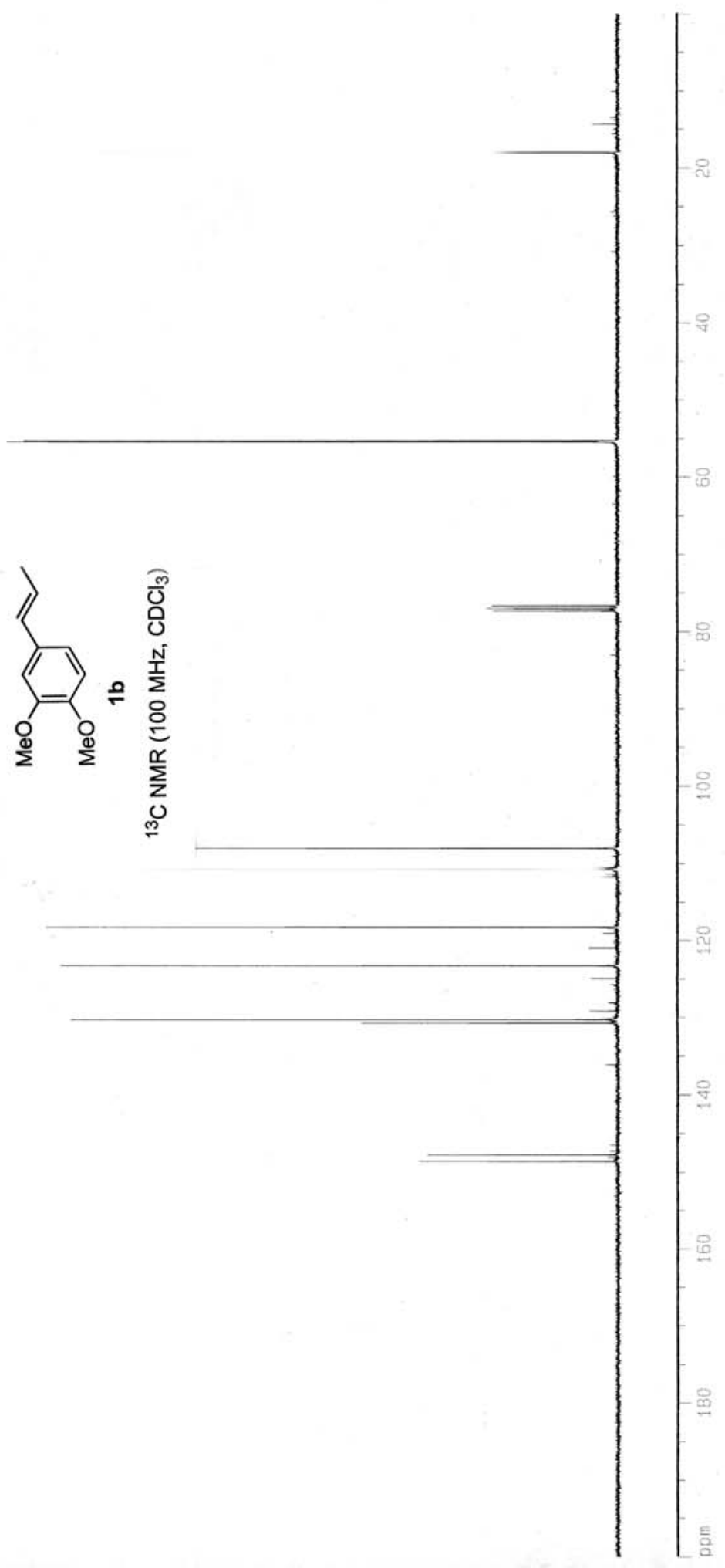




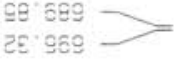

$9 L 900 ;$

$7 \cong \angle 75 ;$ 7E $7905-$ $\mathrm{C7}, \mathrm{90 \textrm {C } -}$ :9 $\angle \angle C Z-$ ex:sizj๘; 9: ९टEट-

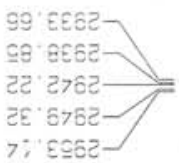
Z.

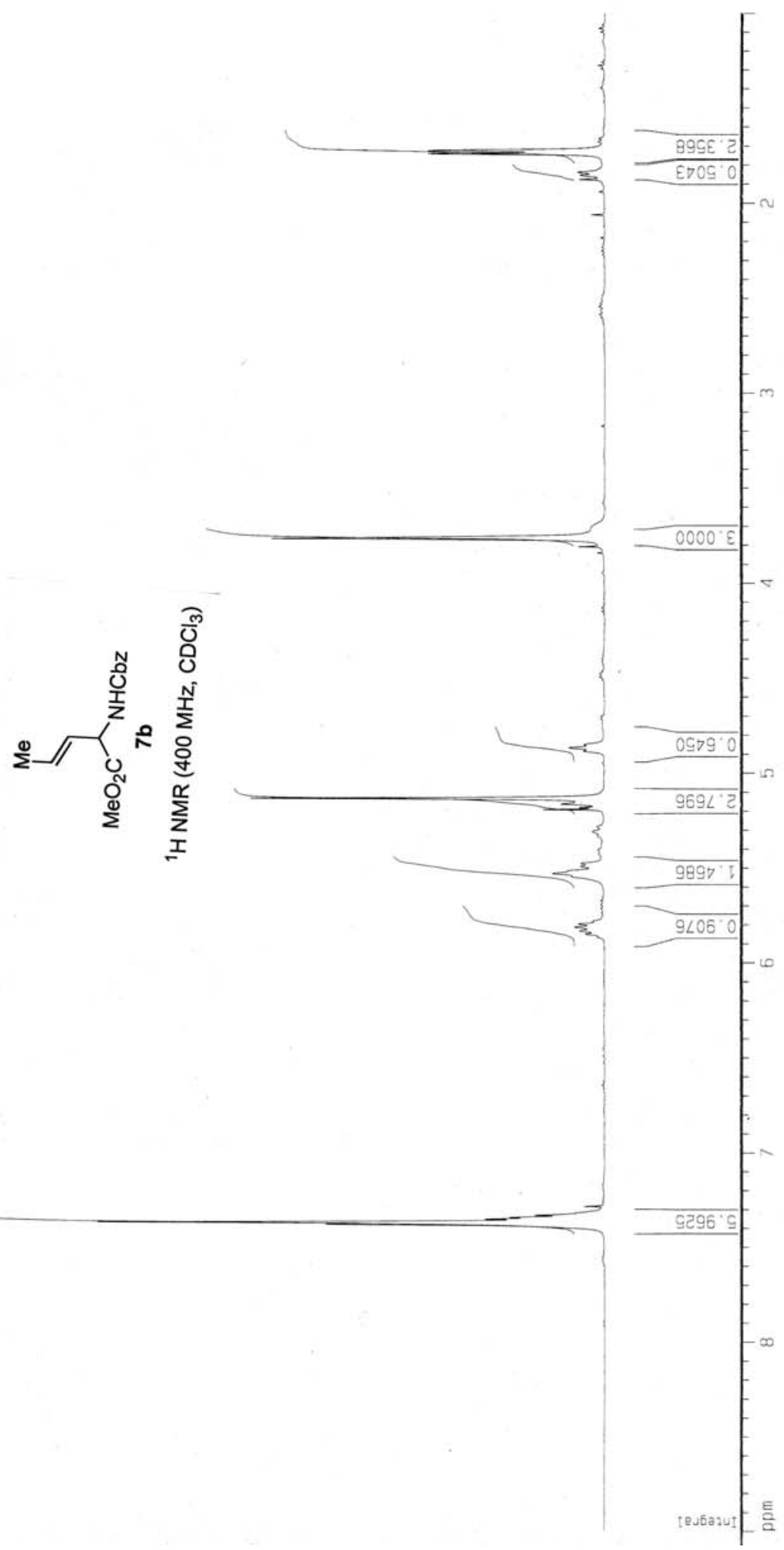




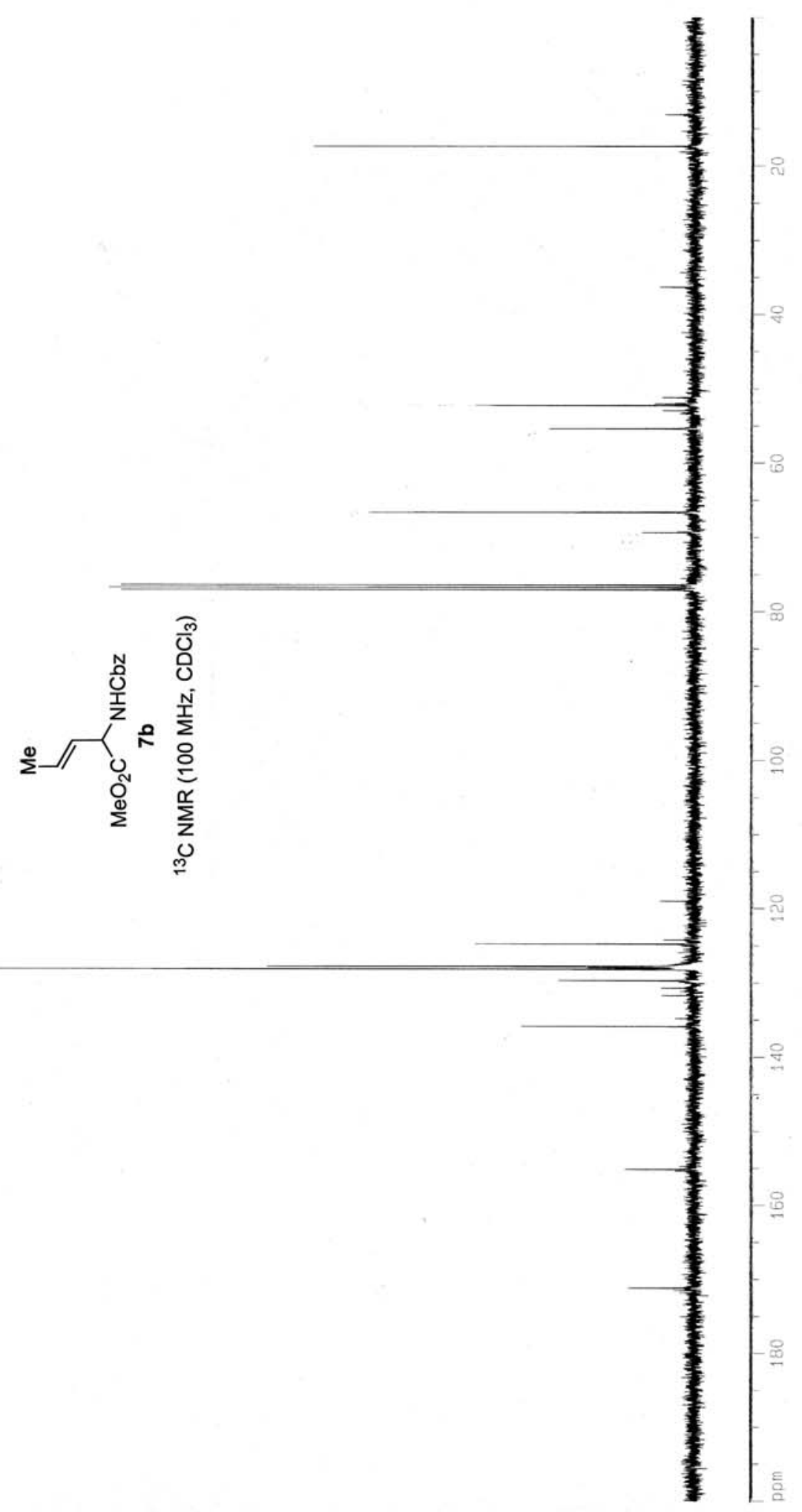




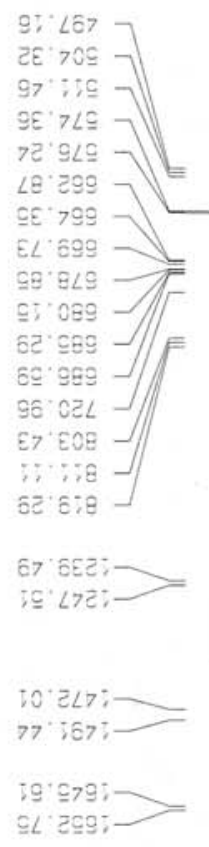

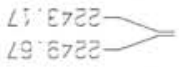

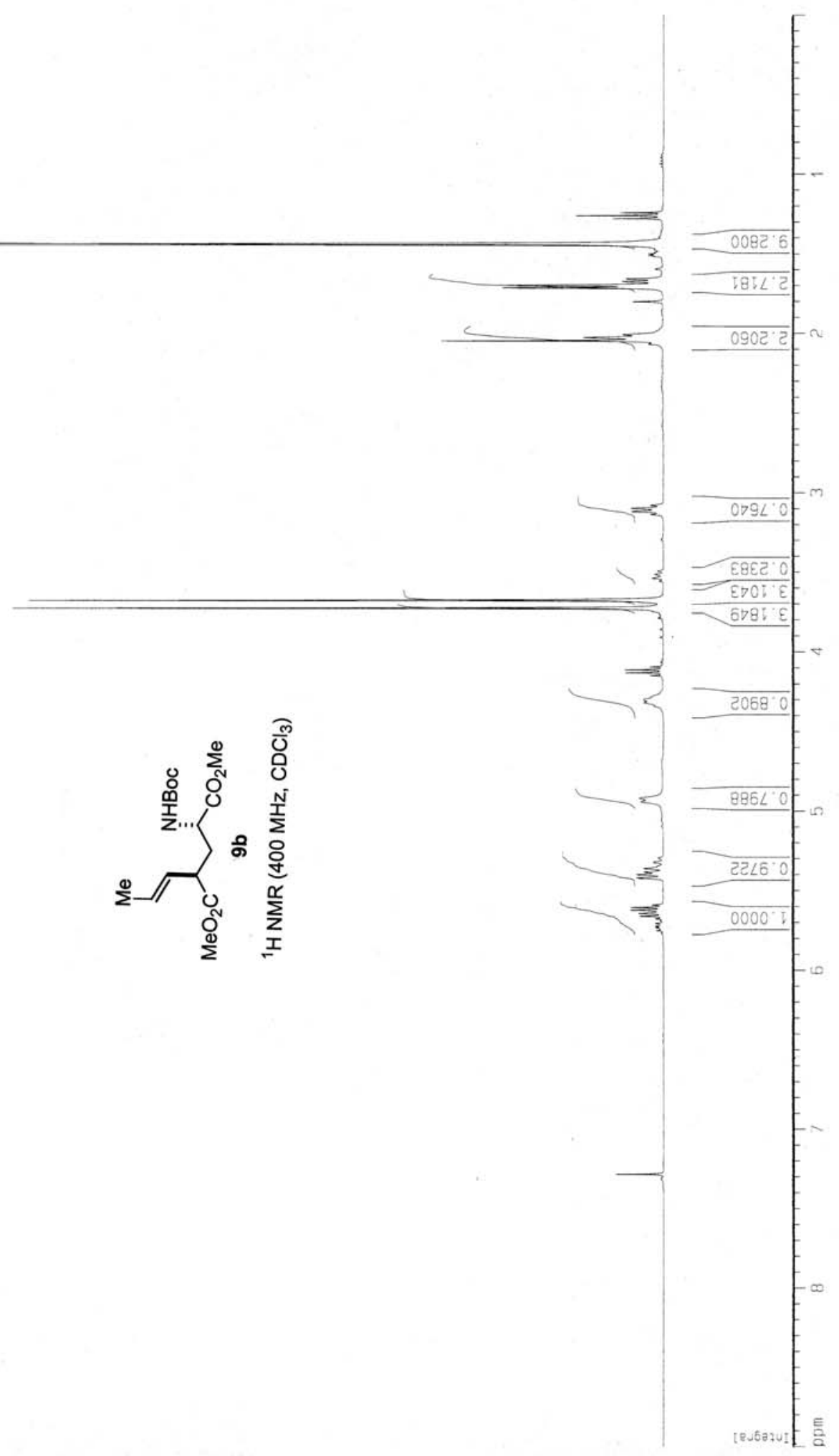




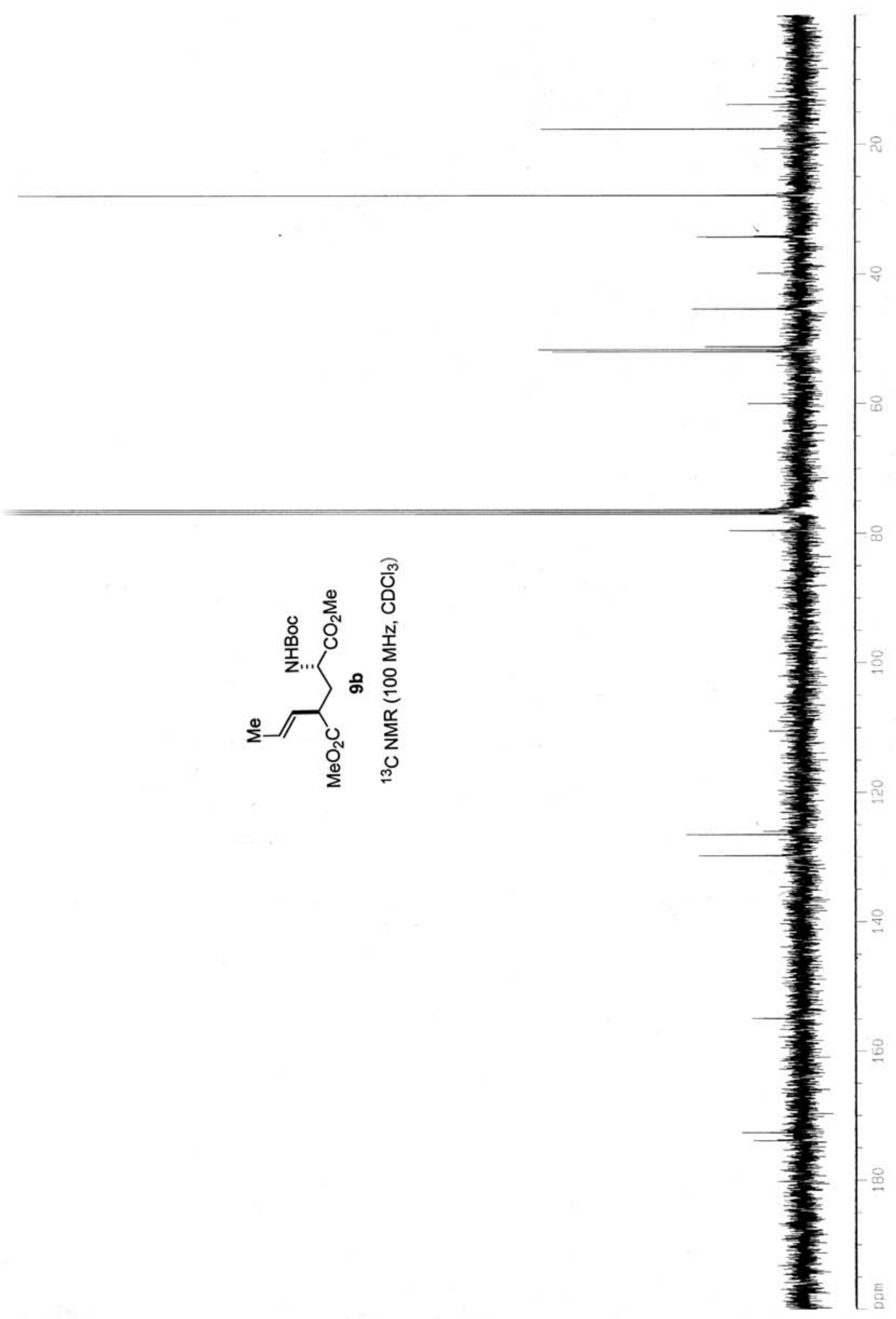




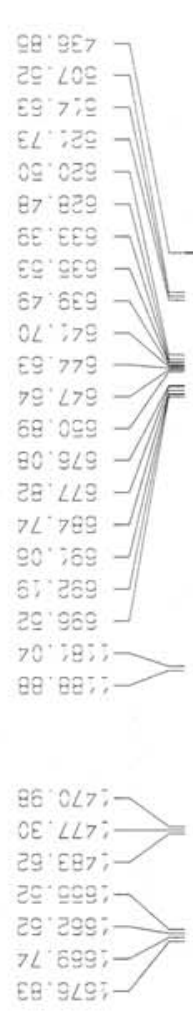

SO $\angle$ L河 LE: $68: z-$

$7 L$. UE:

$\angle E^{\prime} \angle E^{\prime}:$

$i: \varepsilon \overline{c z z}$

हE, รरट5-
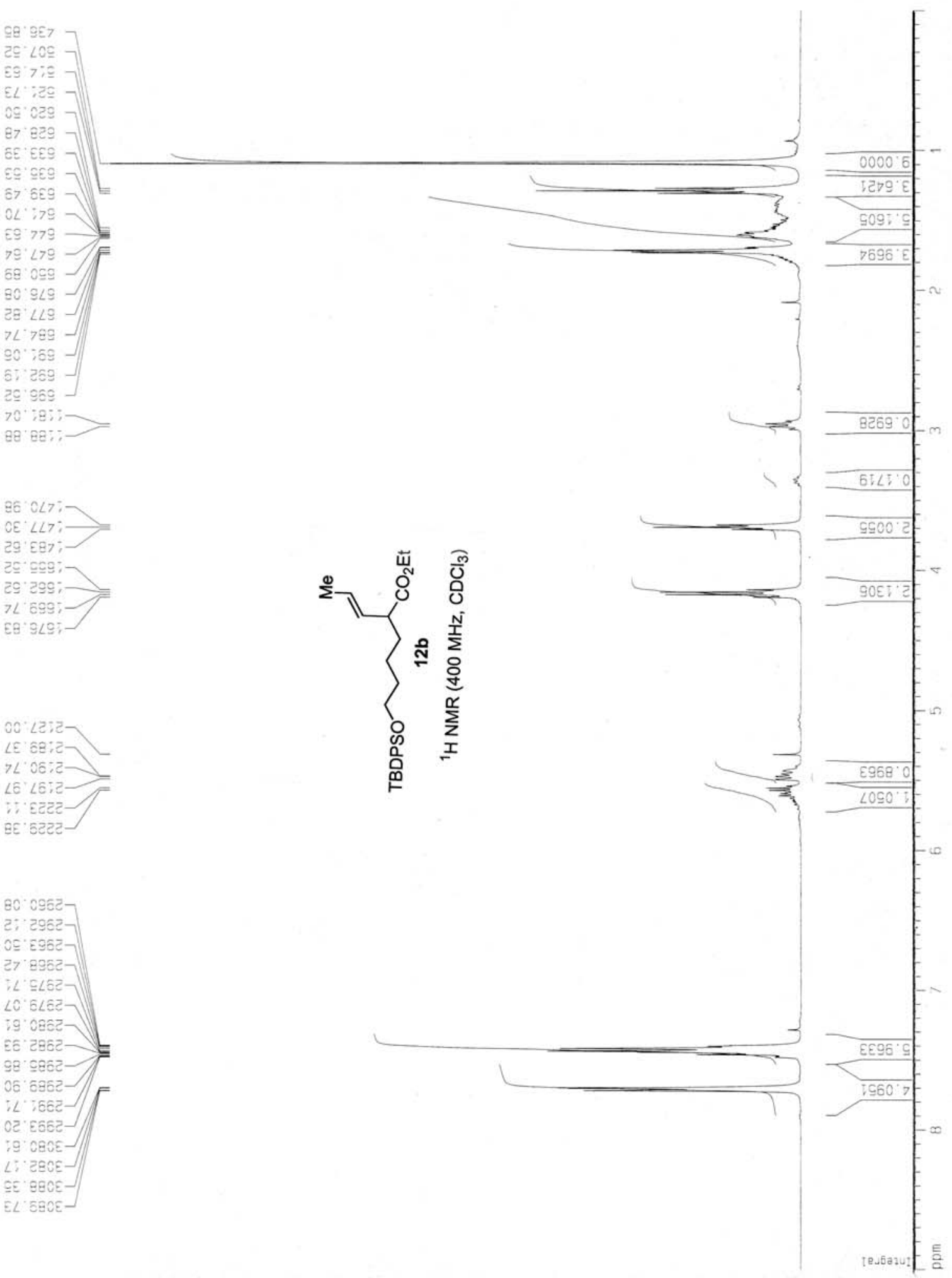


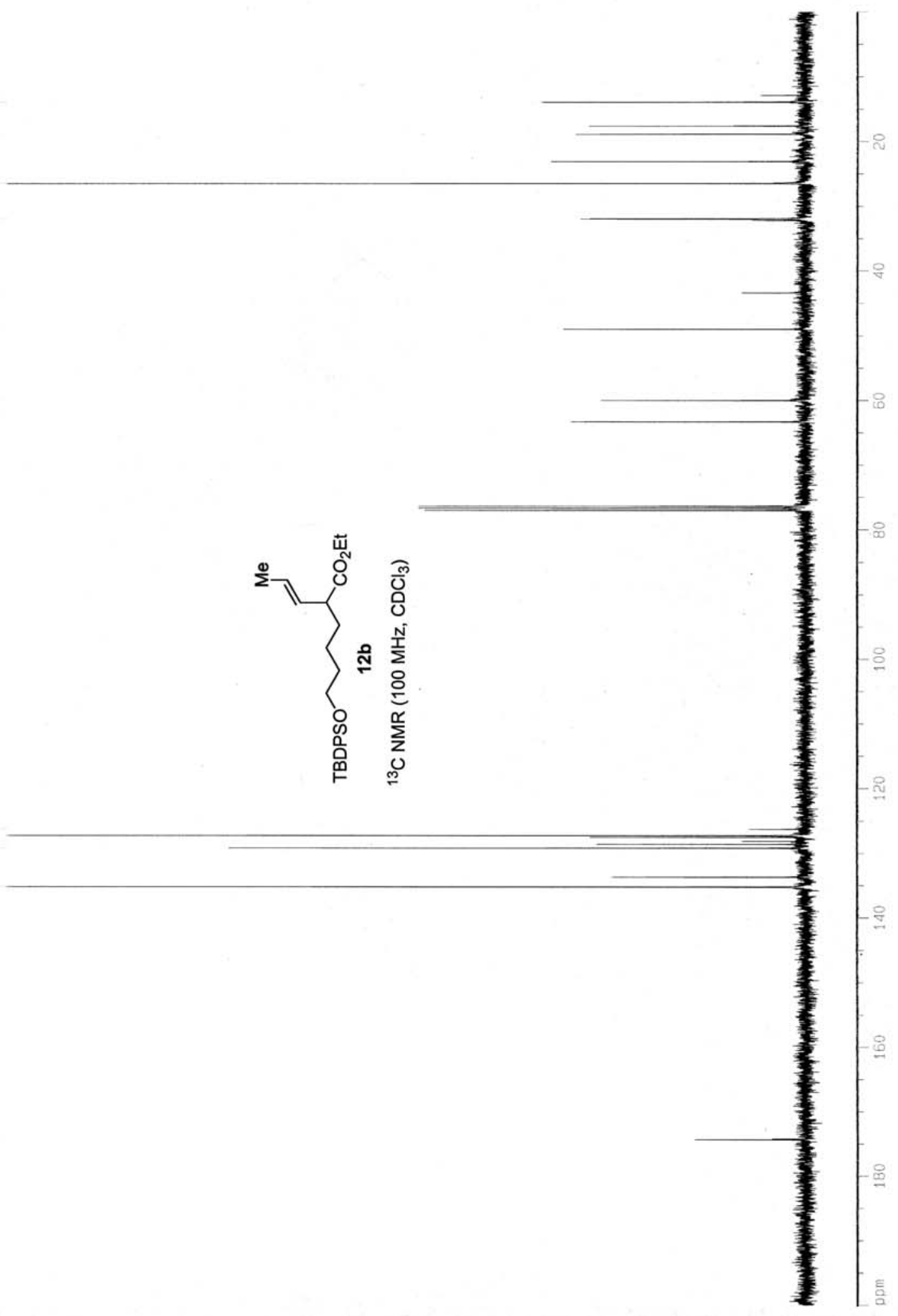




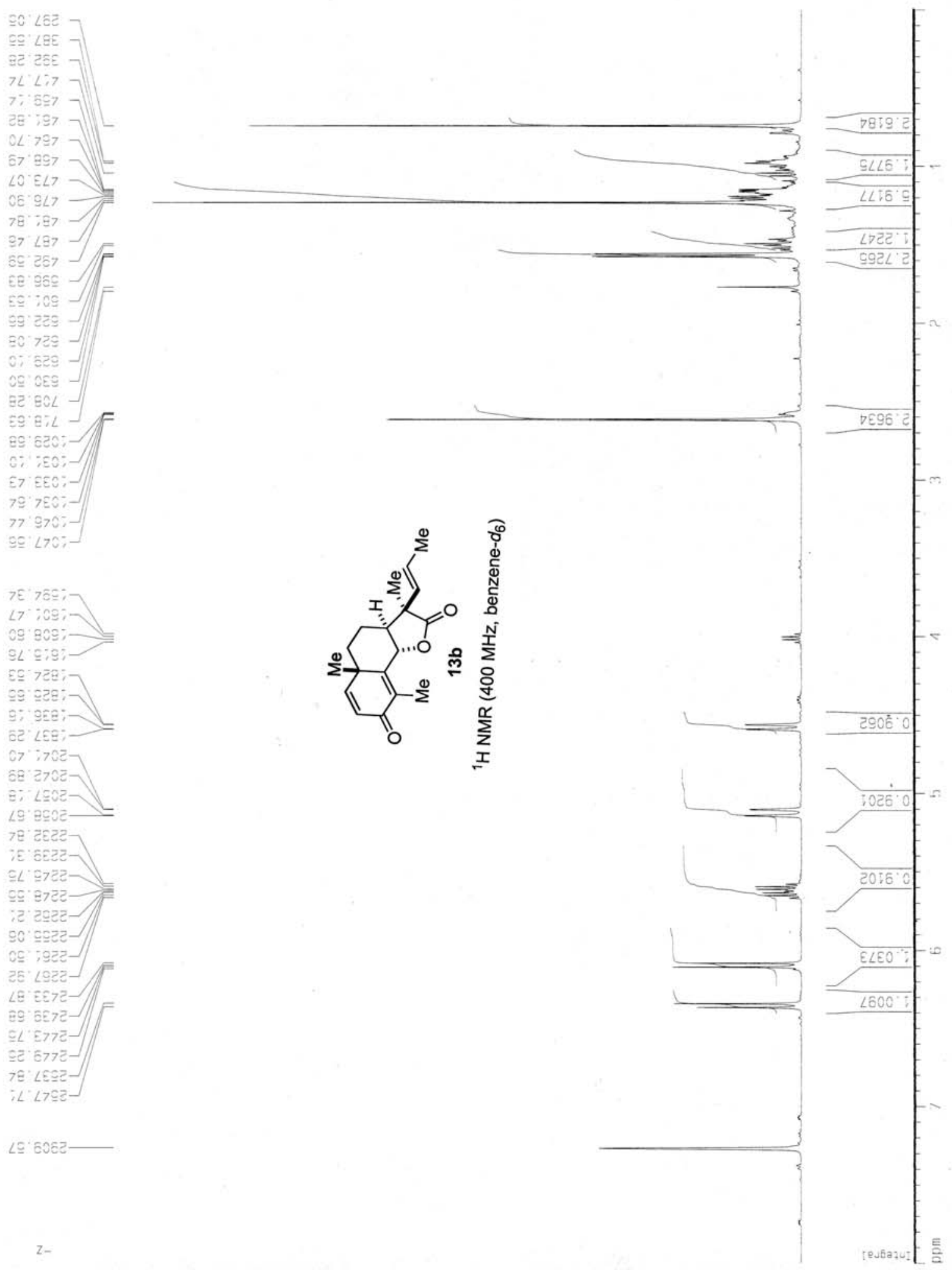




$$
\text { H }
$$




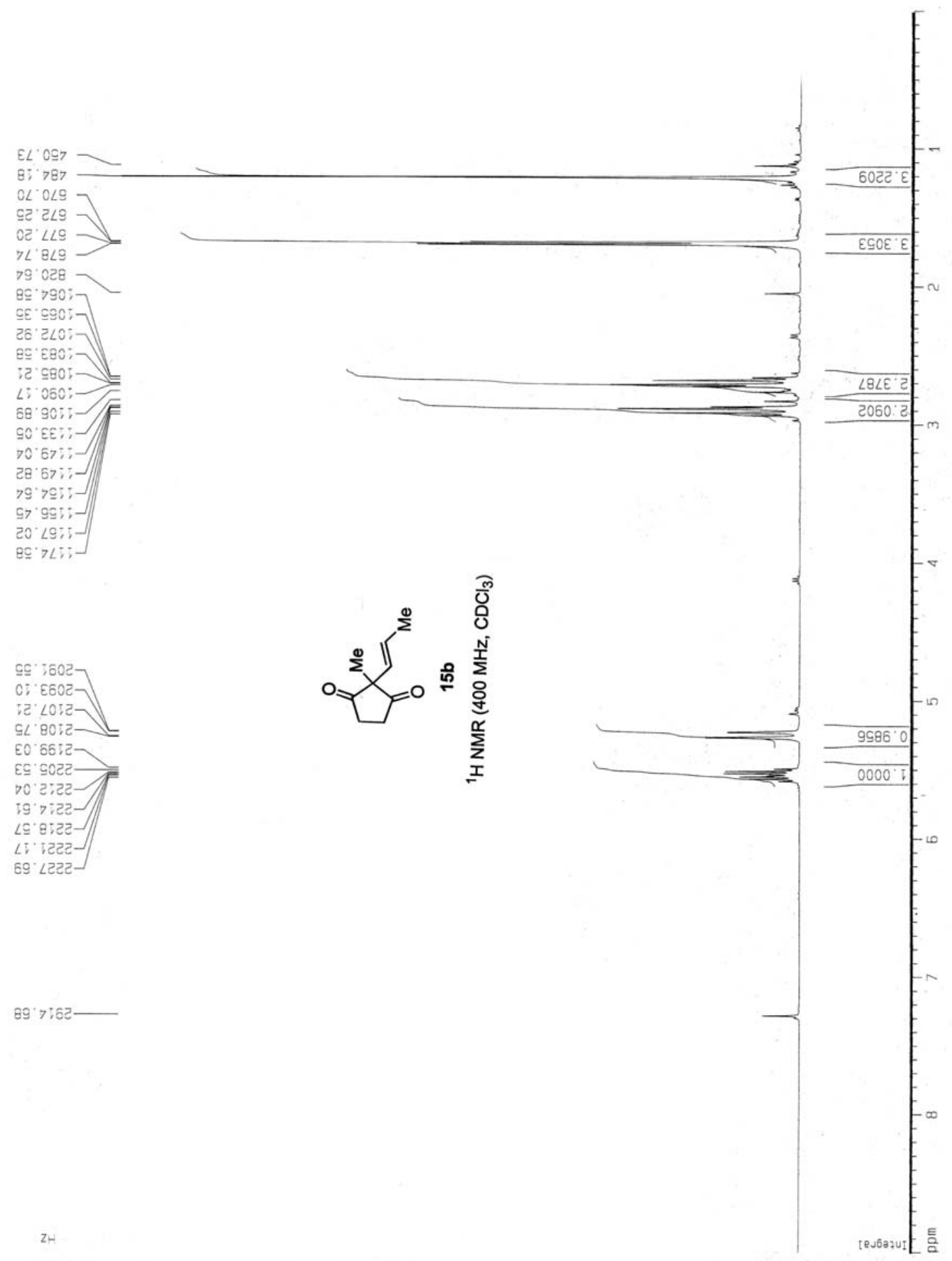




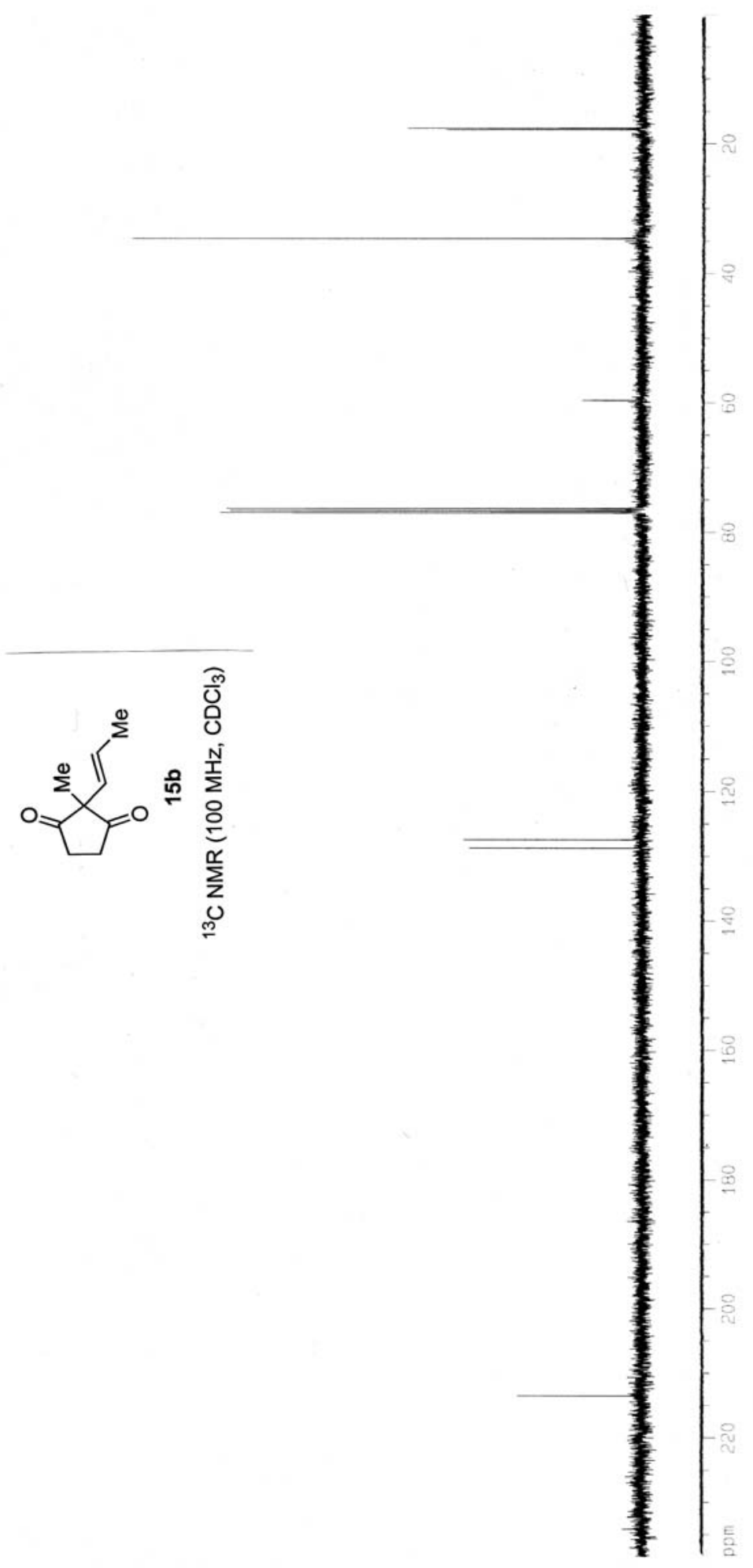



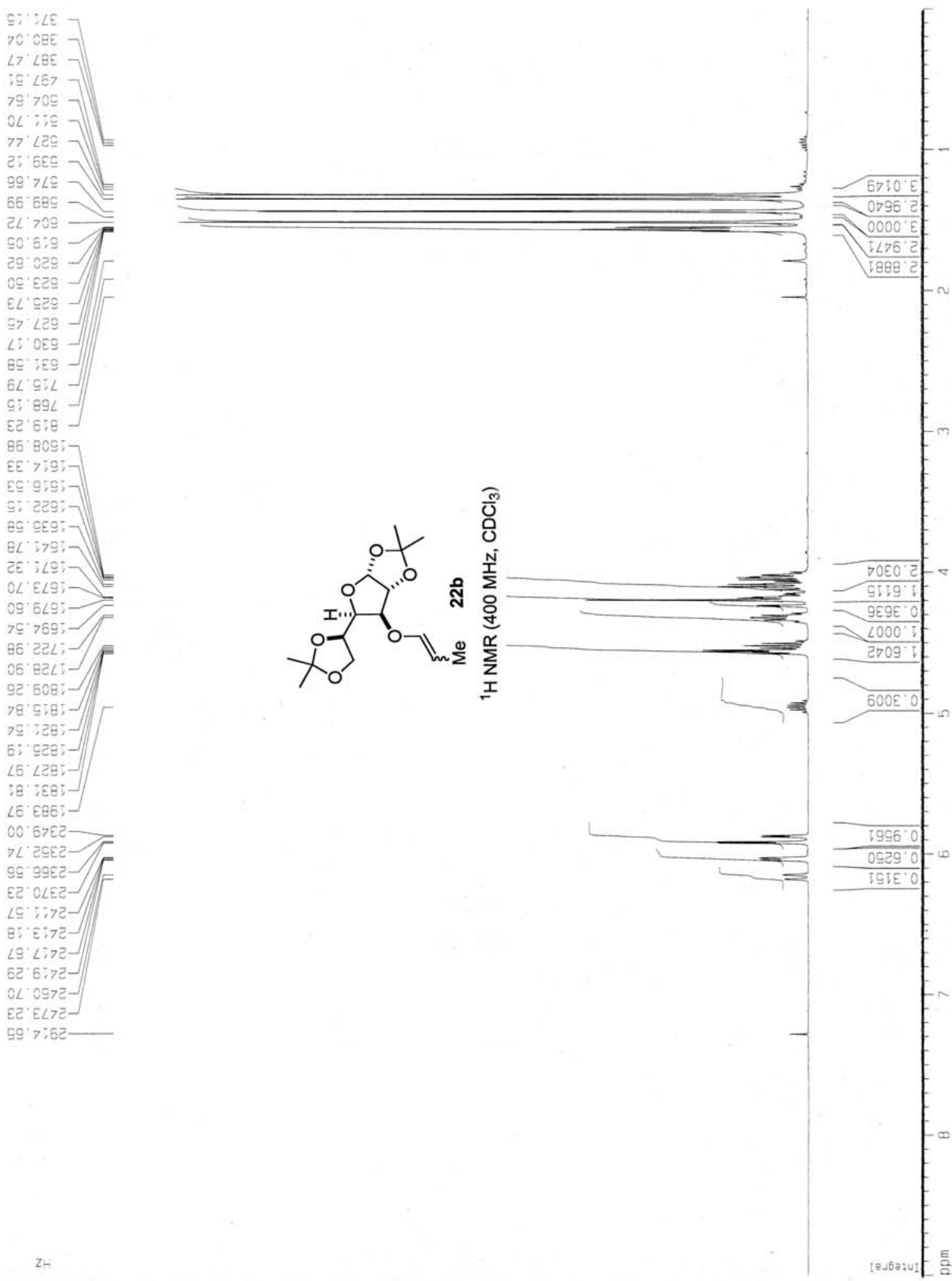

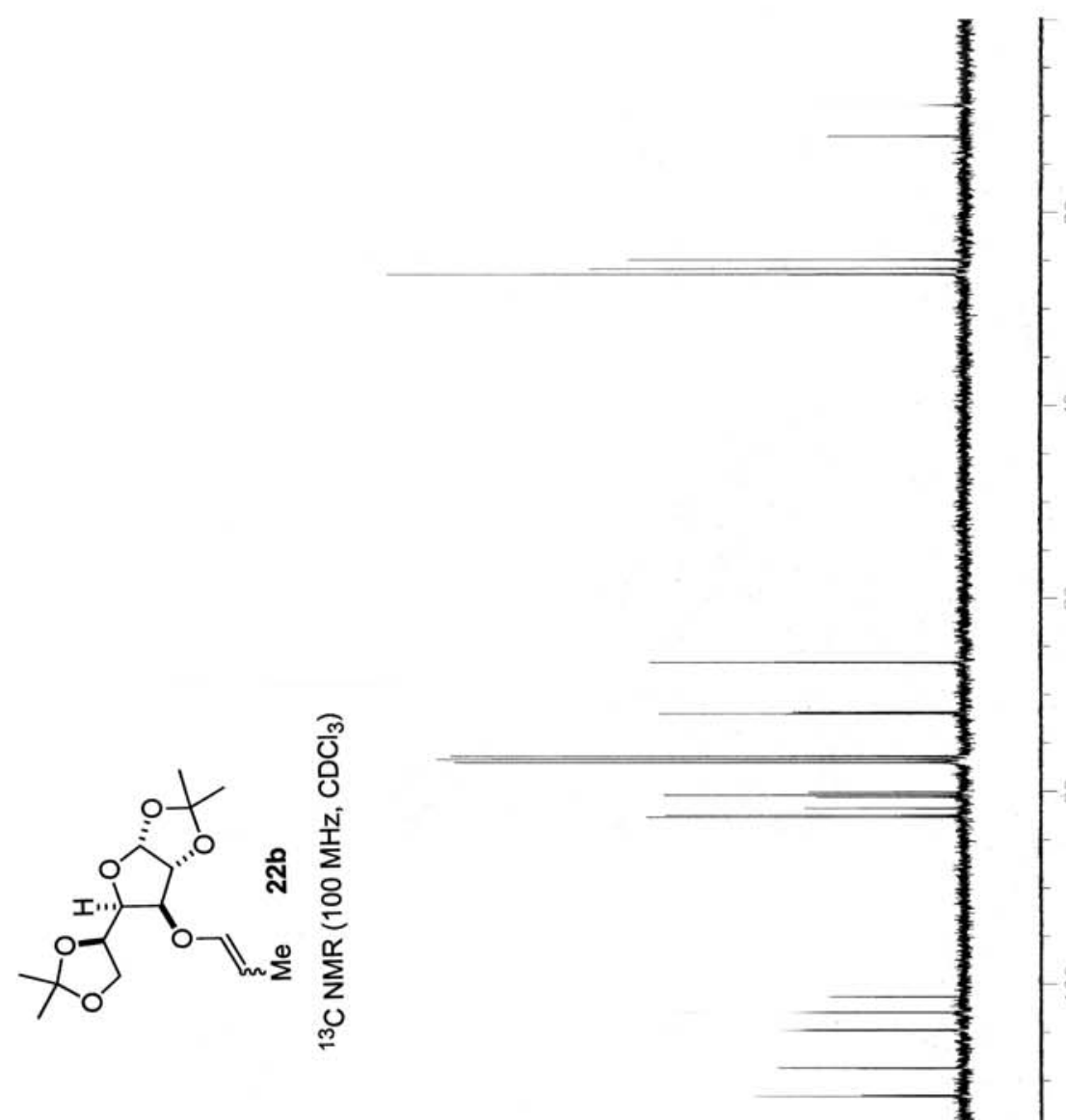

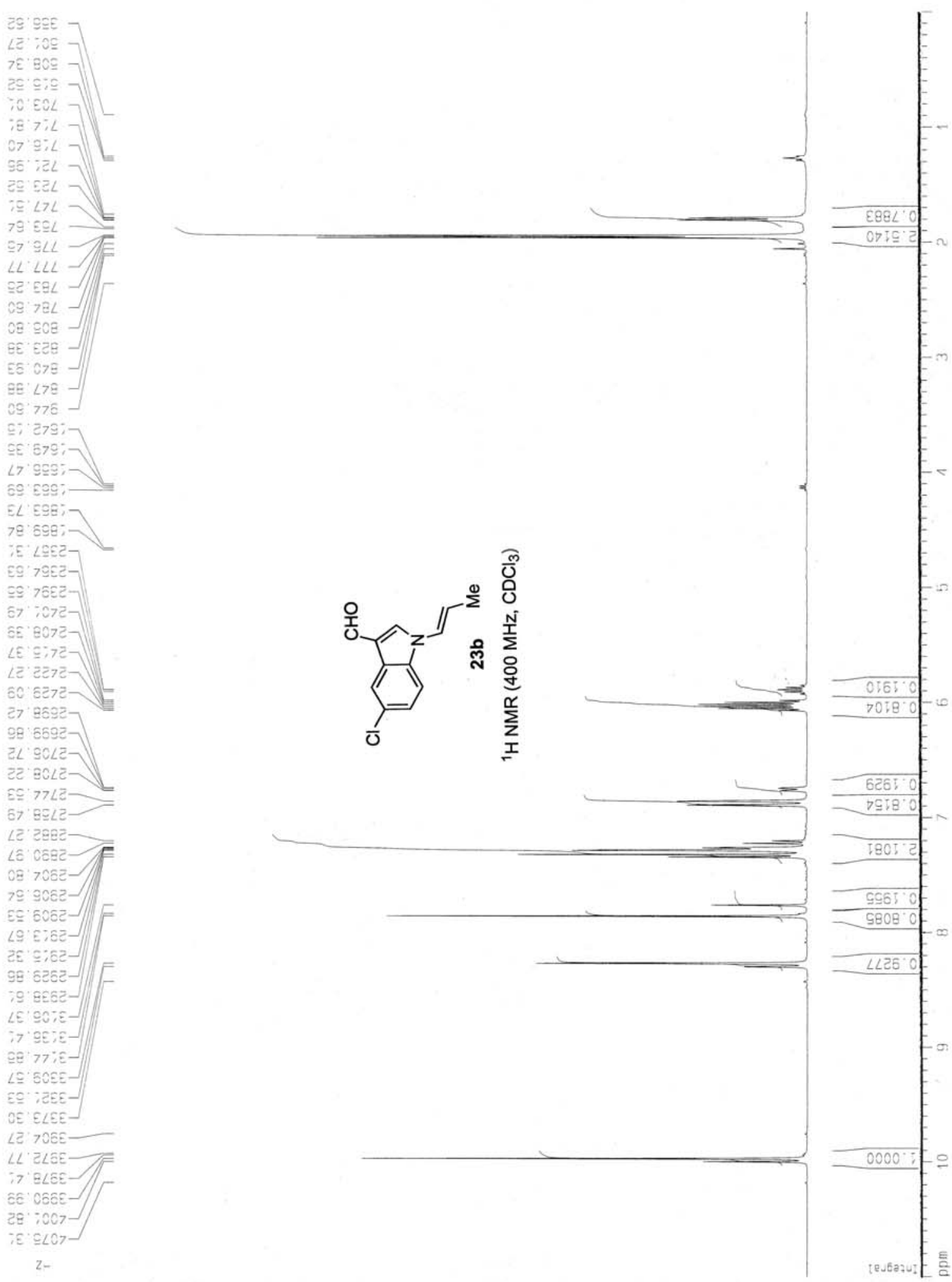


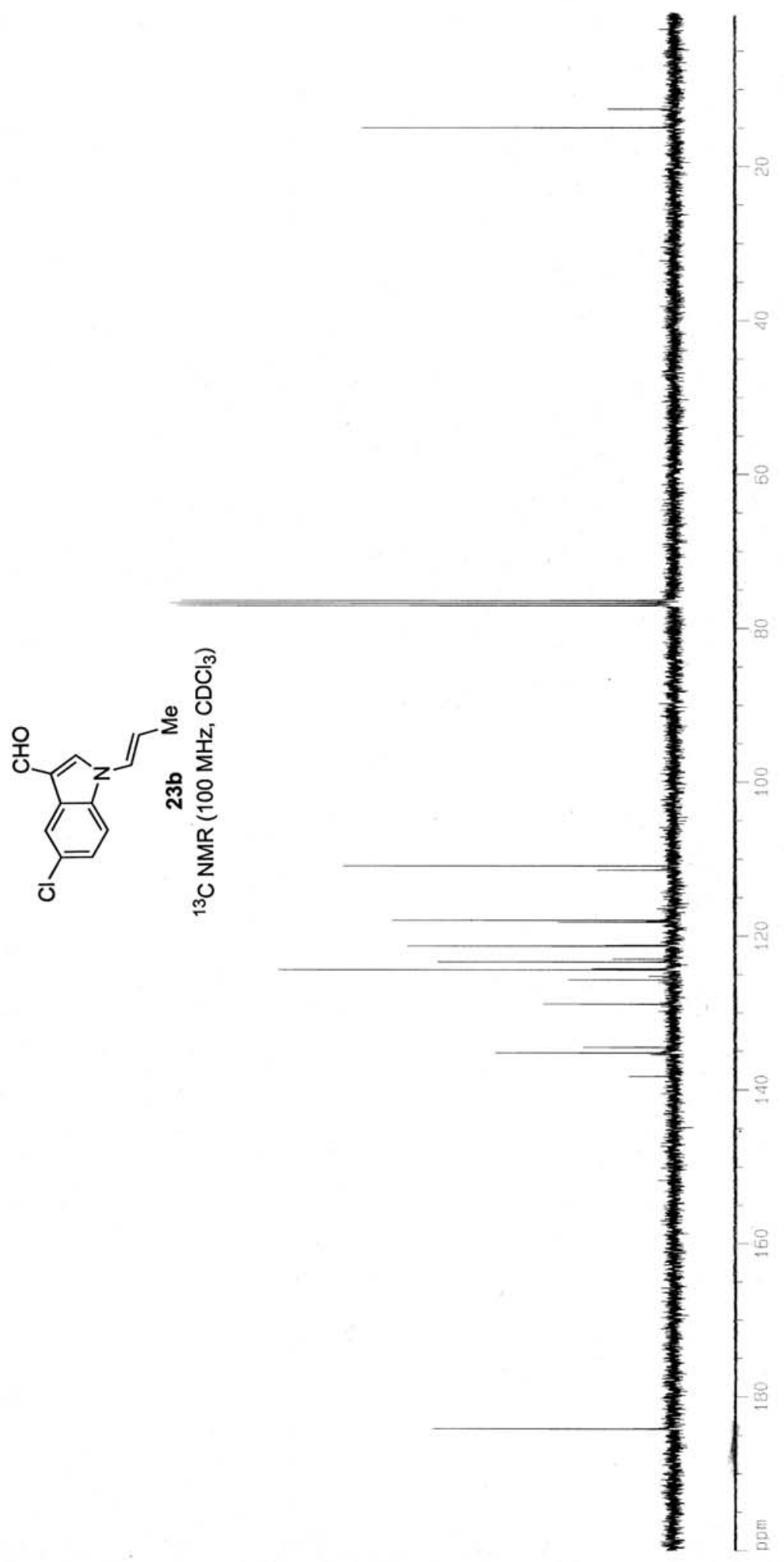




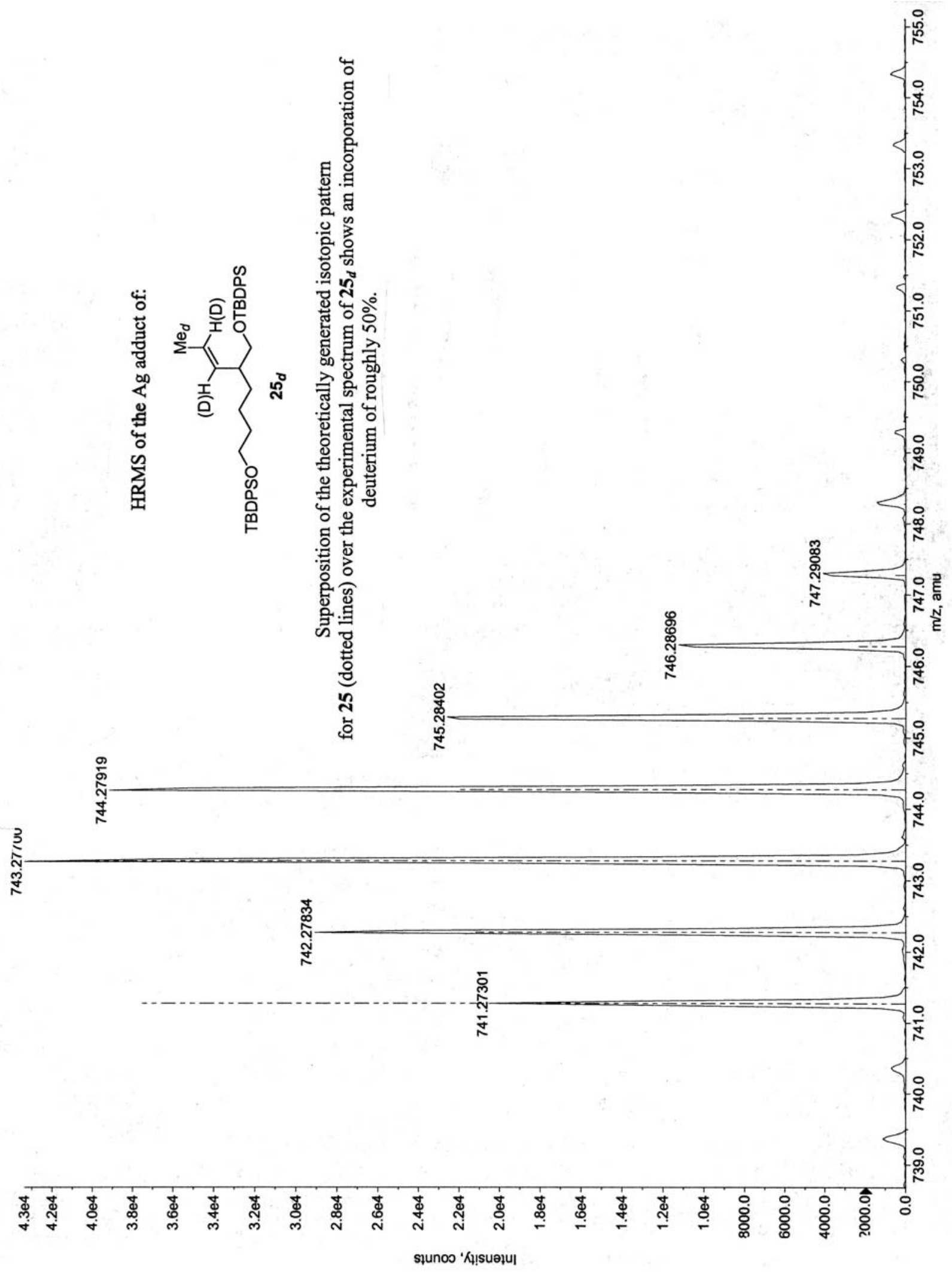

